九州大学学術情報リポジトリ

Kyushu University Institutional Repository

\title{
Zur fossilen Flora aus der Oya-Formation von Kiushiu, Japan
}

Takahashi, Kiyoshi

Faculty of Sciences, Kyushu University

https://doi.org/10.5109/1524117

出版情報：九州大學理學部紀要：Series D, Geology. 5 (1)，pp.47-67，1954-08-30. Faculty of Science, Kyushu University バージョン :

権利関係 : 
Mem., Fac. Sci. Kyushu Univ., Ser. D, Geology, Vol. V. No. 1, pp. 47-67, text figs. 1, 2, plate I-VIII, August, 1954

\title{
Zur fossilen Flora aus der Oya-Formation von Kiushiu, Japan
}

\author{
von
}

\section{Kiyoshi TAKAHASHI}

\section{Einleitung}

Die Ausführungen von Nathorst (1883, 1888) und Fiorin (1920), die die Untersuchungen der känozoischen Pflanzenfossilien behandeln, besonders die der jungtertiären und quartären, sind ausserordentlich wertvoll und spielen in der Geschichte der Paläobotanik eine grosse Rolle. Unter Berücksichtigen der augenblicklichen Formulierung sind die Einteilungen überholt und unsystematisch. Trotz gründlicher Bearbeitung der Pflanzenfossilien in Kiushiu, durch Nathorst und Florin, ist man über das Auftreten von Pflanzen im geologischen Zeitalter $\mathrm{zu}$ keinem endgultigen Resultat gekommen.

Die paläobotanischen, stratigraphischen, und paläoklimatischen Probleme der Fossilien sind in dem letzten Jahren von Dr. S. ENDo, Dr. S. MikI usw. behandelt worden. In dieser epochemachenden Bearbeitung, hat Dr. S. Miki durch die von ihm gesammelten Pflanzenreste die Umgebung ihrer Pflanzenwelt sehr klar gemacht.

In verschiedenen Gegenden in Kiushiu, können wir in den jüngertertiären und quartären Schichten eine grosse Anzahl Pflanzenfossilien feststellen. Ich bin der Auffassung, dass die Pflanzenfossilien wichtiger als Muschelschalenreste für die Altersbestimmung (besonders, der jüngeren Ablagerungen) sind.

Zuerst habe ich die Fossilien aus den verschiedenen Lokalitäten der Ôya-Formation in südlichen Gebiet der Halbinsel Shimabara, Prov. Nagasaki, gesammelt und erforscht.

Die vorliegende Arbeit setzt es sich zum Ziel, diese Fossilien auf ihre Formen nachzuprüfen und das Ergebnis soll diesem geologischen Problem Klärung schaffen.

Die Fossilien bestehen grösstenteils aus Blättern, die übrigen nur aus einer kleinen Anzahl Zapfen, Samen, usw. Um die Zugehörigkeit der systematischen Gattungen und Arten festzustellen, müssen aufmerksame und sorgfältige Forschungen der Fossilien durchgeführt werden, um damit ein klares Ergebnis der paläobotanischen Untersuchungen $z \mathbf{u}$ bekommen. 
Die Untersuchung der gesammelten Fossilien „Ôya-Flora “ hat ergeben, dass sie der „Akashi-Flora“ und „Mogi-Flora“(?) ähnlich sind.

Dr. S. ENDo, Prof. an der Universität Kumamoto, und Dr. S. MıKI, Prof. an der (Stadt-) Universität Ôsaka, haben meine Ergebnisse auf dem Gebiete der Pflanzenfossilienforschung anerkannt, und Dr. T. Matsumoto, Prof. an der Universität Kiushiu, hat mir sehr wichtige Anregungen gegeben. Herr M. INOuE, der die Pflanzenreste mit mir zusammen gesammelt hat, stand mir bei vielen stratigraphischen Fragen mit Rat und Tat zur Seite.

Prof. Dr. S. Endo, Prof. Dr. S. Mrki, Prof. Dr. T. Matsumoto und Herrn M. INOUE möchte ich hiermit meinen herzlichsten Dank aussprechen. Ich liess mir von Frau Ellingsen und Herrn R. TAKahashi das Manuskript durchsehen. Ihnen möchte ich hiermit meinen verbindlichsten Dank aussprechen.

\section{Fundstätte der Pflanzenfossilien und Stratigraphische Verhältnisse}

Das betreffende Gebiet liegt im Süden der Halbinsel Shimabara, und etwa 25 Kilometer südostlich von Mogi, das seit alters paläobotanisch

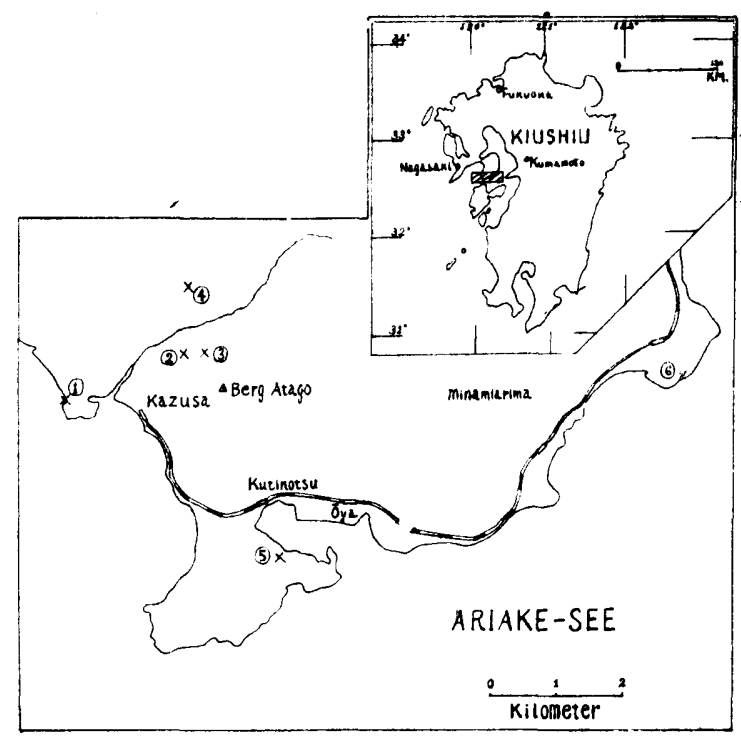

Abb. 1. Die Karte der Stelle und der Fundstätte der Pflanzenfossilien $\mathrm{X}$...Fundort von jüngertertiären Pflanzenfossilien.
(1) Oshima,
(2) Kazusa,
(3) Hasudake,
(4) Kashiyama,
(5) Ôtomari,
(6) Ôe 
bekannt ist. Sechs fossile Fundorte verbreiten sich 10 Kilometer von Osten nach Westen und etwa 4.5 Kilometer von Norden nach Süden.

M. INOUE beschäftigt sich ausschliesslich mit Geologie, insbesondere der Stratigraphie. Die jüngertertiären Ablagerungen stellen sich diskordanzig auf die Basis des ältertertiären Systems in dieser Gegend. Jede Formation der jüngeren Ablagerungen ist aus Ton, Schieferton, Sandstein oder Tuffit zusammengesetzt.

Die Oya-Formation stellt sich diskordanzig auf die Basis des ältertertiären Systems und ist aus Seeablagerungen (?) und Meerablagerungen zusammengesetzt. Man kann die zahlreichenden Pflanzenfossilien aus verschiedenen Schichten an verschiedenen Stellen finden. Einige Insektreste sind auch von mir gefunden worden.

Die pflanzenführenden Ablagerungen von Oshima sind aus Schieferton und Sandstein zusammengesetzt. Die gesammelten Pflanzenreste zeigen den Fortgang des langwierigen Inkohlungsprozesses.

Die pflanzenführende Ablagerung von Kazusa ist Tuffit und der Erhaltungszustand der Fossilien ist verhältnismässig gut.

Die Ablagerung von Hasudake ist Feinsandstein und die Fossilien sind völlig erhalten geblieben.

Die pflanzenführende Ablagerung von Kashiyama besteht aus Tuffit und der Zustand der Fossilien ist verhältnismüssig gut.

Die pflanzenführenden Ablagerungen von ')tomari sind aus Ton und Feinsandstein zusammengesetzt. Einige Reste der gesammelten Pflanzenfossilien werden langwierig karbonisiert.

Die Ablagerungen von Oe bestehen aus Schieferton und Sandstein. Der Zustand der Fossilien ist nicht sehr gut.

In den letzten Jahren haben F. TAKAI und M. INoue in der ÒyaFormation die Reste von Elephantenzähnen, Stegodon orientalis OsBo :N und Elephas sp., gefunden.

\section{List der fossilen Pflanzen}

Erklärung der Kürzungszeichen.

Reste:

Z: Zapfen, S: Samen, B: Blatt, Sb: Schrimblatt,

D: Dorn, F: Frucht und Fruchtbecher

Zahl :

$\mathrm{m}$ : mehr, $\mathrm{g}$ : gemein, $\mathrm{r}$ : rar

Verbreitung der Art oder der am nächsten verwandten Art der Jetztzeit :

NA: Nordamerika, C: China, K: Korea, M: Mandschurei,

F : Formosa, L: Liukiuinseln, J J Japan

Lokalitäten :

1-Oshima, 2-Kazusa, 3-Hasudake, 4-Kashiyama, 5-Ôtomari, 6-Ôe 


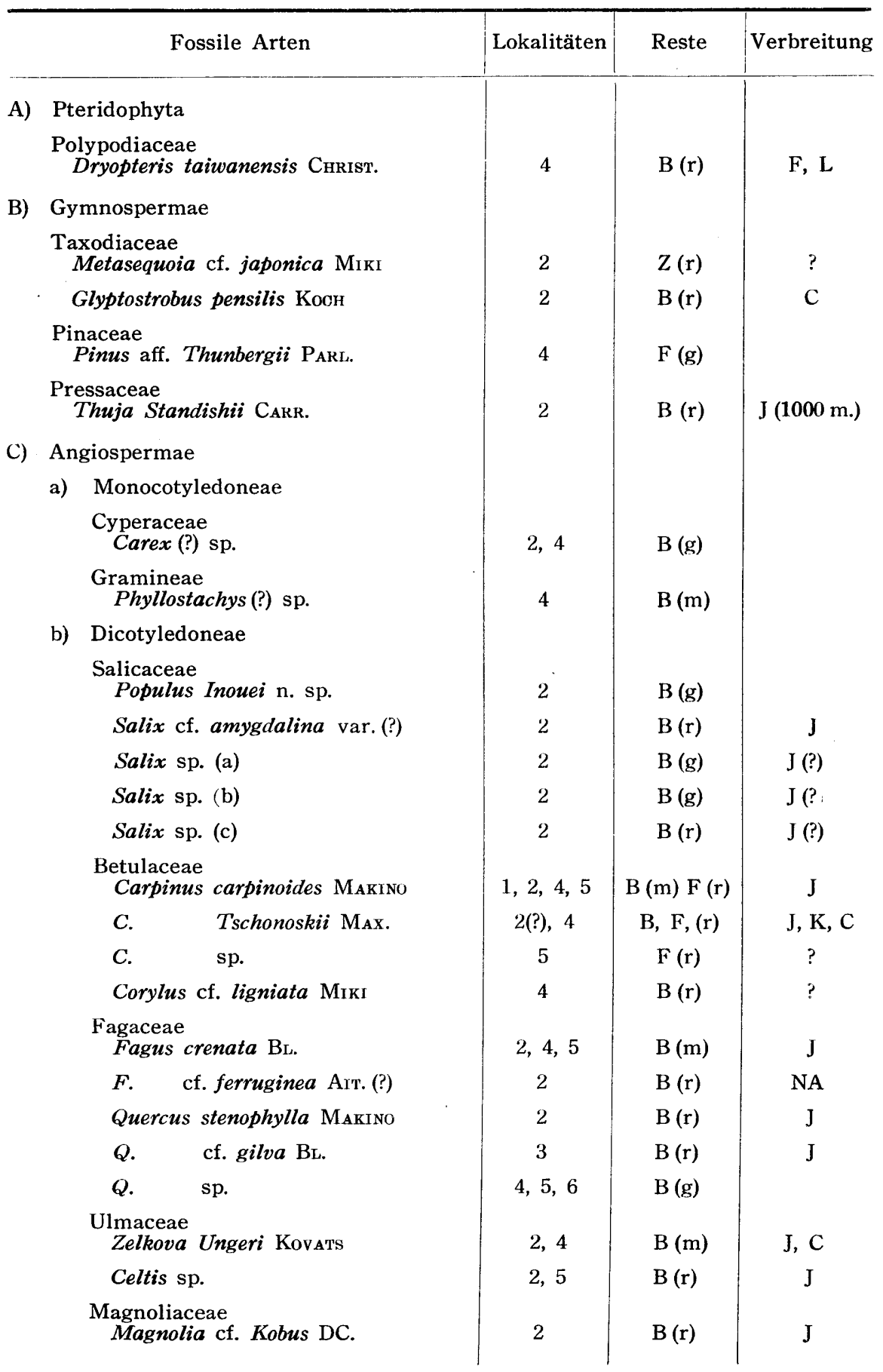




\begin{tabular}{|c|c|c|c|}
\hline Fossile Arten & Lokalitäten & Reste & Verbreitung \\
\hline $\begin{array}{l}\text { Lauraceae } \\
\text { Cinnamomum cf. Camphora SIEs. }\end{array}$ & 4 & $\mathrm{~B}(\mathrm{r})$ & $\mathbf{J}$ \\
\hline sp. & 1 & $B(r)$ & ? \\
\hline $\begin{array}{l}\text { Hamamelidaceae } \\
\text { Liquidambar cf. formosana HANCE }\end{array}$ & 2,5 & $B(r)$ & $\mathrm{F}, \mathrm{C}$ \\
\hline $\begin{array}{l}\text { Rosaceae } \\
\quad \text { Rosa multiflora THUNB. }\end{array}$ & 2 & $\mathrm{~B}, \mathrm{D},(\mathrm{m})$ & $\mathbf{J}$ \\
\hline Rosa sp. & 4 & $\mathrm{~B}(\mathrm{~g})$ & \\
\hline $\begin{array}{l}\text { Leguminosae } \\
\quad \text { Wistaria cf. brachybotys } \mathrm{S} \text {. et Z. (?) }\end{array}$ & 2 & $\mathrm{~B}(\mathrm{r})$ & $\mathbf{J}$ \\
\hline Entada formosana KANEHIRA & 2 & $B(m)$ & F (1000 m.) \\
\hline $\begin{array}{l}\text { Euphorbiaceae } \\
\text { Sapium sebiferum Rox. var. } \\
\quad \text { pleistoceaca MIKI }\end{array}$ & 2 & $\mathrm{~S}(\mathrm{~m})$ & $\mathrm{C}, \mathrm{M}$ \\
\hline $\begin{array}{l}\text { Buxaceae } \\
\text { Buxus japonica MuELE }\end{array}$ & $2,3,4,5$ & $B(m)$ & $\mathrm{J}(1000 \mathrm{~m})$. \\
\hline $\begin{array}{l}\text { Aceraceae } \\
\quad \text { Acer pictum ThUNB. }\end{array}$ & 2,4 & $B(r)$ & $\mathrm{J}$ \\
\hline A. palmatum ThUnв. & 4,5 & $B(r)$ & $\mathrm{J}$ \\
\hline A. cf. parviflorum F. et S. (?) & 2,3 & $F(r)$ & $\mathbf{J}$ \\
\hline A. cf. diabolicum BL. (?) & 2 & $F(r)$ & $\mathbf{J}$ \\
\hline $\begin{array}{l}\text { Sapindaceae } \\
\quad \text { Xanthoceras cf. sorbifolia BGE. }\end{array}$ & 2 & $B(r)$ & $\mathrm{C}$ \\
\hline $\begin{array}{l}\text { Rhamnaceae } \\
\text { Paliurus nipponicus MIKI }\end{array}$ & 2 & $\mathrm{~S}(\mathrm{~m})$ & $\mathrm{C}, \mathrm{M}$ \\
\hline $\begin{array}{l}\text { Tiliaceae } \\
\quad \text { Tilia cf. japonica Simk. }\end{array}$ & 4 & $\mathrm{Sb}(\mathrm{r})$ & $\mathbf{J}$ \\
\hline $\begin{array}{l}\text { Theaceae } \\
\text { Camellia japonica L. }\end{array}$ & 2,4 & $\mathrm{~B}(\mathrm{~g})$ & $\mathrm{J}, \mathrm{C}, \mathrm{L}$ \\
\hline Stewartia cf. monadelpha S. et Z. & 2 & $B(r)$ & J \\
\hline $\begin{array}{l}\text { Liliaceae } \\
\quad \text { Smilax China L. }\end{array}$ & 2,4 & $\mathrm{~B}(\mathrm{~m})$ & $\mathrm{J}, \mathrm{L}, \mathrm{F}$ \\
\hline $\begin{array}{l}\text { Ebenaceae } \\
\text { Diospyros Kaki THUNB. }\end{array}$ & 4 & $B(r)$ & $\mathbf{J}$ \\
\hline
\end{tabular}




\section{Beschreibung der Arten}

\section{A) PTERIDOPHYTA \\ Polypodiaceae}

Dryopteris taiwanensis CHRIST.

Tafel I, Fig. 1.

Das vorliegende unvollständig erhaltene Fiederblatt zeigt den offenbaren Nervenverlauf.

Die Fiederblattchen sind etwas lanzettförmig, etwa $5 \mathrm{~mm}$. lang, und etwa $2.5 \mathrm{~mm}$. breit. Die Seitennerven sind einfach, und erreichen fast den Rand.

Das vorliegende Farnblatt stimmt unzweifelhaft mit der in Liukiuinseln und Formosa lebenden Art Dryopteris taiwanensis CHRIST, überein.

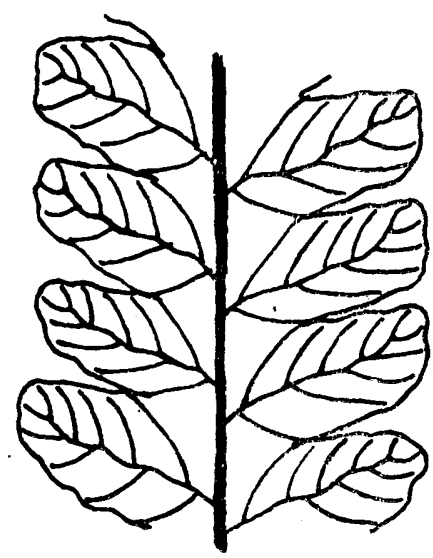

Textfig. 1. Der Nervenverlauf des Farnblattes. Fast 4 mal vergrössert.

\section{B) GYMNOSPERMAE}

\section{Taxodiaceae}

\section{Metasequoia cf. japonica MIKI}

Tafel I, Fig. 2a, b

Sequoia japonica Endo: (1936) S. 172, Fig. 5, 7-13; (1939) S. 337, Taf. 23, Fig. 15. Metasequoia japonica Miki:. (1941) S. 262, Taf. 5 D, Fig. 8 Ab, H.

Zwei Zapfen, die in dem Tuffitlager bei Kazusa gefunden wurden, gehören der gegenständigen Metasequoia-Zapfenform an. Die vorliegenden Zapfenfragmente stimmen wahrscheinlich mit den von Endo als Sequoia japonica beschriebenen Pflanzen überein, die später von MrKI als Metasequoia japonica beschrieben worden sind.

Die äussere Form der Zapfen ist fast kugelförmig. Ihre Länge zeigt etwa $15 \mathrm{~mm}$. und die Breite $15 \mathrm{~mm}$. Zwölf Zapfenschuppen sind kreuzgegenüberstehend auf der Achse und ihre Höhe zeigt etwa $4 \mathrm{~mm}$.

\section{Glyptostrobus pensilis $\mathrm{KocH}$}

Tafel 1, Fig. 4.

Glyptostrobus europaeus HeER: ENDo u. OKUTsu (1936) S. 138, Fig. 1-3; Endo (1939) S. 336, Taf. 1, Fig. 14.

Glyptostrobus pensilis Косн: Mrki (1941) S. 259, Taf. 5 H, Fig. 7 C-F. 
Ich fand einzigen $Z$ weig in der Tuffitschicht bei Kazusa.

Der vorliegende Zweig stimmt unzweifelhaft mit der von ENDo und OKuTsu als Glyptostrobus europaeus beschriebenen Pflanzen überein, die später von MıKI als Glyptostrobus pensilis beschrieben worden sind.

\section{Pinaceae}

Pinus aff. Thunbergii PARL.

Tafel I, Fig. 5 a-f.

Ich fand einige Samenreste in der Tuffitschicht bei Kazusa.

Die vorliegenden Samen gehören unzweifelhaft zu Pinus durch die morphologischen Merkmale.

Sie sind verkehrt-eiförmig. Ihre Länge schwankt zwischen 5 und 6 $\mathrm{mm}$., und die Breite zwischen 3.5 and $4.5 \mathrm{~mm}$. Der Samenflügel ist $8 \mathrm{~mm}$. lang und $5 \mathrm{~mm}$. breit.

Die Grösse der Samen gleicht beinahe mit der von Pinus densiflora S. et Z. und Pinus Thunbergii PARL. In der Grösse des Flügels sind aber sie kleiner als letztere beiden, deren Flügeln im allgemeinen dreimal so lang als ihre Samen sind.

\section{Cupressaceae}

Thuja Standishii CARRIERE

Tafel I, Fig. 3.

Thuites sp.: Nathorst (1888) S. 31, Taf. 9, Fig. 19.

Thujopsis-dolabrata S. et Z.: Mгкі (1936) Fig. 1 D.

Thuja japonica Max.: MIKI (1937) S. 308, Fig. 1 D.

Beim Zerspalten eines Stückes kam das kleine Fragment zum Vorschein.

Der vorliegende blattartige Zweig gleicht vielmehr der heutigen lebenden Art Thuja Standishii CARR. als der anderen lebenden Art Thujopsis dolabrata S. et Z. im morphologischen Merkmal, welches die kurzschuppigen Seitenblätter ist.

C) ANGIOSPERMAE

a) MONOCOTYLEDONEAE

Cyperaceae

Carex (?) sp.

Tafel I, Fig. 6a, b.

Die systematische Bestimmung der Monokotyledonenreste ist im allgemeinen sehr schwierig. Da ferner die fossilen Blätter meistens nur in Bruchstücken gefunden wurden, wird die Bestimmung noch unsicherer. 
Aus dem Grunde habe ich die vorliegenden Blattfragmente nur als Carex bezeichnet, dass sie einen ähnlichen Bau wie die zu dieser Gattung gebrachten Reste zeigen.

\section{Gramineae}

Phyllostachys (?) sp.

Tafel I, Fig. 7 a, b, c.

Die vorliegenden Blätter sind verhältnismässig vollkommen erhalten. Es ist aber unmöglich, dass mit Sicherheit dieselben bestimmen. Sie gehören wahrscheinlich zu Phllostachys.

\section{b) DICOTYLEDONEAE}

\section{Salicaceae}

Populus Inouei n. sp.

Tafel I, Fig. 12 a, b.

Einige Blätter sind breit-eiförmig, am Rande grosswellenförmig gezähnt, etwa $40 \cdot \mathrm{mm}$. lang, und $36 \mathrm{~mm}$. breit. Ihre Basis ist rundartig oder breitkeilartig.

Die betreffenden Reste sind ähnlich der in Japan lebend Art Populus Sieboldi Mig., und der Zahn unterscheidet sich aber von ihm der letztere in Form.

\section{Salix cf. amygdalina L. var.(?)}

Tafel I, Fig. 8.

Salix amygdalina L. var.: MIKI (1933) S. 621, Taf. 1, Fig. 4 H; (1937) S. 310, Fig. 30-P; (1941) S. 263, Fig. 9 A, B.

Das vorliegende Blatt ist unvollständig erhalten, aber es gehört wahrscheinlich zu Salix amygdalina L. var.

\section{Salix sp. (a)}

Tafel I, Fig. 9 a, b, c.

Einige Blätter sind linienartig, am Rande fein gezähnt, $3-4 \mathrm{~cm}$. lang under $0.8-1 \mathrm{~cm}$. breit.

Die betreffenden Blätter gehören unzweifelhaft zu Salix, aber die weitere Bestimmung ist unmöglich.

Salix sp. (b)

Tafel I, Fig. 10 a, b.

Einige Blätter sind linienartig, am Rande fein gezähnt, 5-6 cm. lang und $0.9-1.1 \mathrm{~cm}$. breit.

Diese Blätter gehören mit Sicherheit zu Salix, aber die weitere Bestimmung ist unmöglich. 
Salix sp. (c)

Tafel I, Fig. 11.

Das vorliegende Blatt ist $\mathrm{zu}$ unvollständig, um sicher bestimmt werden zu können.

Dieses etwas lederartige Blatt gehört unzweifelhaft zu Salix.

\section{Betulaceae}

\section{Carpinus carpinoides MAKINO}

Tafel II, Fig. 1-10; Tafel III, Fig. 1-5.

Carpinus subjaponica Nath.: Nath. (1888) S. 32, Taf. 9, Fig. 12-15.

C. japonica BL.: ENDo (1933) S. 40, Fig. 4; (1940) S. 55, Taf. 6, Fig. 4, 8, 11, 14, 18; Taf. 8, Fig. 18; Taf. 9, Fig. 14 .

C. carpinoides Makino: Miki (1941) S. 267, Fig. 10 C-F.

Die zahlreichen Blätter und einige Fruchtbecher wurden in den Tuffitund Tonschichten bei Kazusa und Kutinotsu gefunden.

Die Blätter und der vollkommen erhaltene Fruchtbecher, Fig. 9, stimmen vollständig mit denen von Carpinus carpinoides MaKino überein. Bei Fig. 10 ist die Spitze nicht erhalten und der Fruchtbecher kurzig gestielt.

\section{Carpinus Tschonoskii MAX.}

Tafel II, Fig. 11; Tafel III, Fig. 6.

Carpinus subyedoensis Kon'no: (1931) S. 145, Taf. 7, Fig. 1.

C. Tschonoskii Max.: Miki (1938a) S. 232, Fig. 12 D; S. 236, Fig. 14 B; (1941) S. 268, Fig. 10 D, E.

Einziges Blatt und einziger Fruchtbecher, welche auf den Steinplatten liegen, stimmen vollständig mit denen von Carpinus Tschonoskii Max. überein. Das erstere, das auf Taf. III, Fig. 6 abgebildet ist, ist etwa lanzettförmig, $6 \mathrm{~cm}$. lang, und $2.4 \mathrm{~cm}$. breit. Der Blattrand zahnt scharf doppeltsägeförmig. Der letztere, der auf Taf. II, Fig. 11 abgebildet ist, zeigt $17 \mathrm{~mm}$. lang, $6 \mathrm{~mm}$. breit, und die unsymmetrische Form mit den viermal ausgekerbten Zähnen an einer Seite.

\section{Carpinus sp.}

Tafel II, Fig. 12.

Das auf Taf. II, Fig. 12 abgebildete Stück stellt ein Fragment eines Fruchtbechers dar, dessen vermutete Form, Grösse, und Nervenverlauf der chinesischen Art Carpinus Tungtzeensis Hu ähnlich sind. Eine sichere Bestimmung des Exemplares ist aber bei seiner Unvollständigkeit nicht möglich.

Corylus cf. ligniatus MrkI

Tafel IV, Fig. 9 a, b.

Corylus ligniatus MıкI: MıkI (1941) S. 268, Fig. $10 \mathrm{H}$. 
Einige Blätter sind elliptisch, länger gespitzt, am Rande doppeltig gezähnt, $7.1 \mathrm{~cm}$. lang und $2.8 \mathrm{~cm}$. breit.

Die betreffenden Blätter stimmen unzweifelhaft mit der von MrKI beschriebenen Art Corylus ligniatus Mrki überein.

\section{Fagaceae}

\section{Fagus crenata BluME}

Tafel III, Fig. 7-12; Tafel IV, Fig. 1-8.

Fagus sylvatica L.: NAтH. (1888) S. 33, Taf. 9, Fig. 1, 2; S. 37, Taf. 12, Fig. 6-10.

F. $\quad$ sylvatica L. var. asiatica DC.: NATH. (1888) S. 37, Fig. 1, 2, 4.

F. $\quad$ sylvatica L. var. Sieboldi EndL. Kolwai (1915) S. 44, Fig. 1-4.

F. $\quad$ crenata BL.: Endo (1931) S. 18, Fig. 12; (I933) S. 43, Fig. 14; (1940) S. 57, Taf. 6, Fig. 2, 3, 6, 9, 13, 16, 20, 23, 24;; Taf, 12, Fig. 4 b.

F. crenata BL.: Mıкі (1937) S. 313, Fig. 11; (1938 a) S. 229, Fig. 10 I.

Die vorliegenden zahlreichen Blätter sind eiförmig, kurz gestielt, und am Rande etwas gebucht. Ihre Länge schwankt zwischen 42 und $62 \mathrm{~mm}$., und die Breite zwischen 23 und $55 \mathrm{~mm}$. Der Blattgrund ist rundlich oder breit-keilartig. Der Primärnerv zeigt den Zickzack an dem oberen Teil. Die Sekundärnerven, welche jederseits 7-10 vorhanden sind, laufen gegen die Buch $z \mathrm{u}$, und erreichen dieselbe aber nicht, sondern sich fast rechtwinklig aufwärts biegen. Zuweilen kann der Blattrand auch kleine Zähne zeigen.

Die vorliegenden Blätter von Kutinotsu and Kazusa stimmen vollständig mit der heutigen lebenden Art Fagus crenata BL. bei den oben erwähnten Merkmalen überein.

Fagus cf. ferruginea ArT.(?)

Tafel V, Fig. 1.

Fagus ferruginea A1т.: NarH. (1883) S. 43, Taf. 4, Fig. 11-24; Taf. 5, Fig. 1-11; Taf. 6, Fig. 1.

F. ferruginea АІт.: Мıкі (1941) S. 270, Fig. 11 B, Cc.

Das vorliegende Blatt ist zu unvollständig, um mit Sicherheit bestimmt werden zu können, gehört wahrscheinlich zu Fagus ferruginea AIT. durch die morphologischen Merkmale.

\section{Quercus stenophylla MAKINO}

Tafel V, Fig. 6.

Quercus stenophylla Makino: MIKI (1941) S. 270, Fig. 12 E, F.

Der vorliegende Blattrest ist lanzettförmig und am Oberrande fein gezähnt. Seine Länge ist etwa $65 \mathrm{~mm}$. und seine Breite $22 \mathrm{~mm}$.

Dieses Blatt stimmt mit der jetzt in Japan lebenden Art Quercus stenophylla MAKINo überein. 
Quercus cf. gilva BL.

Tafel. V, Fig. 5.

Quercus gilva BL.: Mrki (1938 a) S. 219, Fig. 4 N-P; (1941) S. 270, Fig. 12 G, H.

Dieses Blatt ist unvollständig erhalten, um vollkommen bestimmt werden zu können, gehört aber wahrscheinlich zu Quercus gilva BL.

Das Blatt hat kleine Zähne am Oberrande und seine Breite zeigt 20 $\mathrm{mm}$.

\section{Quercus sp.}

Tafel V, Fig. 2, 3, 4.

Einige Blattreste sind so fragmentarisch, dass sie nicht mit Sicherheit bestimmt werden können. Da aber auch sie die quercusartigen Zähne zeigen, so müssen sie zu Quercus gehören.

\section{Ulmaceae \\ Zelkova Ungeri Kovats \\ Tafel V, Fig. 8 a-g.}

Planera Ungeri Етт.: NATH. (1888) S. 7, 9, Taf. 1, Fig. 5, (7-11).

Zelkova sp.: Mrкi (1933) S. 623, Taf. J, Fig. A, B.

Z. Ungeri Kovats: Miki (1937) S. 312, Taf. 9 N, 0, Fig. 3 D, E; (1938 a) S. 219 , Fig. 4 L; S. 224, Fig. 6 B; (1941) S. 272, Fig. 12 I.

Die zahlreichen Blätter wurden in den Tuffit- und Tonschichten bei Kutinotsu und Kazusa gefunden.

Die vorliegenden Blätter sind lanzettförmig oder eiförmig, etwa $3 \mathrm{~mm}$. gestielt und am Rande tief gezähnt. Ihre Länge schwankt zwischen 15 und $20 \mathrm{~mm}$. und die Breite zwischen 6 und $10 \mathrm{~mm}$. Die Blattspitze ist kurz. Die Sekundärnerven, die jederseits 7-9 vorhanden sind, laufen gegen den Zahn zu. Der Tertiärnerv verlauft gegen die nächste Bucht aus dem Sekundärnerv fast rechtwinklig abwärts.

Im fossilen Zustande ist diese Art in den Tertiärablagerungen Europas, Asiens und Nordamerikas nachgewiesen. Sie lebt nicht jetzt in Japan.

\section{Celtis sp.}

Tafel V, Fig. 7.

Das unvollständig erhaltene Blattfragment zeigt den für Celtis sehr charakteristischen Nervenverlauf. Die betreffenden Reste sind aber zu unvollständig, um mit Sicherheit ihre Arten zu bestimmen.

\section{Magnoliaceae}

Magnolia cf. Kobus DC.

Tafel V, Fig. 9.

Magnolia Kobus DC.: MiKI (1937) S. 315, Fig. 4 B; $(1938$ a) S. 216, Fig. 2 H; S. 224, Fig. 6 N; S. 233, Fig. 12 J; S. 237, Fig. 14 Db; (1941) Fig. 13 B. 
Das betreffende Blatt ist verkehrt-eiförmig, etwa $75 \mathrm{~mm}$. lang und 40 $\mathrm{mm}$. breit. Es hat die kurze Spitze und die etwas scharfe Blattbasis.

Der vorliegenden Rest, der den schöhen Abdruck nicht hinterlässt, stimmt wahrscheinlich mit der heutigen lebenden Art Magnolia Kobus DC. überein.

\section{Lauraceae}

Cinnamomum cf. Camphora SiEB.

Tafel VI, Fig. 2.

Das kleine unvollständig erhaltene Blatt ist vermutlich eiförmig, ganzrandig, und lederartig.

Die Leitbündelverlauf ist gefiedert und das etwas schwache Leitbündelpaar tritt aus der Nähe der Basis des die Mitte des Blattes durchziehenden Leitbündels aus.

Das betreffende Blatt stimmt wahrscheinlich mit der lebenden Art Cinnamomum Camphora SieB. überein.

\section{Cinnamomum sp.}

Tafel VI, Fig. 3.

Das vorliegende Blatt ist $z \mathfrak{u}$ unvollständig, um sicher bestimmt werden $z u$ können, gehört aber unzweifelhaft zu Cinnamomum durch ein Leitbündelpaar aus der Nähe der Basis des Blattes.

\section{Hamamelidaceae}

\section{Liquidambar cf. formosana HANCE}

Tafel VI, Fig. 4.

Liquidambar formosana HANCE: NATH. (1883) S. 55, Taf. 8, Fig. 6-9; (1888) S. 24, Taf. 6, Fig. 14, 15; KRYstofovich (1920) S. 9, Taf. 2, Fig. 3, 4; Ko'No (1931) Taf. 4, Fig. 1; Taf. 12, Fig. 7; Endo u. Morita (1932) S. 47, Taf. 6, Fig. 1-10; Taf. 7, Fig. 1-8; MıкI (1941) S. 275, 276, Taf. 6 A, B, Fig. 14 A-C.

Diese vorliegenden Blätter sind $z u$ unvollständig erhalten, um sicher bestimmt werden zu können, gehören aber unzweifelhaft zu Liquidambar durch drei strahligen Leitbündel und den fein gezähnten Rand, und wahrscheinlich zu der jetzt in Mittel- und Südchina und Formosa lebenden Art Liquidambar formosana HANCE.

\section{Rosaceae}

\section{Rosa multiflora THUNB.}

Tafel VI, Fig. 5 a-m.

Rosa polyantha S. et Z.: Mгкі (1937) S. 316, Fig. 5 F; (1938 a) S. 237, Fig. 14 I.

Ich fand zahlreichen Zweige, Dörner und Blätter in der Tuffitschicht bei Kazusa. 
Die betreffenden Reste stimmen unzweifelhaft mit der in Japan lebenden Art Rosa multiflora Thunb. durch die Formen des Dornes und Blattes überein.

\author{
Rosa sp. \\ Tafel VI, Fig. 6 a, b, c.
}

Einige kleinen Blätter sind leider zu unvollständig, um sicher bestimmt werden zu können. Die betreffenden Blätter gehören unzweifelhaft $z u$ Rosa. Sie scheinen mit dem Blatt von Rosa akashiensis übereinzustimmen, sind aber nicht sicher zu bestimmen.

\title{
Leguminosae
}

Wistaria cf. brachybotys S. et Z. (?)

Tafel VI, Fig. 7 a, b.

Ich fand einige Blätter in der Tuffitschicht bei Kazusa.

Die Blätter sind eiförmig, ganzrandig, $40 \mathrm{~mm}$. lang und $24 \mathrm{~mm}$. breit. Der Blattstiel ist etwa $3 \mathrm{~mm}$. Die Seitennerven sind jederseits 9 vorhanden.

Beim ersten Anblick haben diese Blätter einige Ähnlichkeiten mit dem Blatt von Wistaria ligniata MrkI, aber gleichen nicht in der Form der Blattspitze und in der Länge des Stiels.

\section{Entada formosana KANEHIRA}

Tafel VI, Fig. 1 a-i.

Entada formosana KANEHIRA: TANAI (1952) S. 233, Taf. 22, Fig. 6, 7.

Ich fand die zahlreichen Blattreste in der Tuffitschicht bei Kazusa.

Die vorliegenden Blattreste sind elliptisch, ganzrandig und etwa unsymmetrisch. Ihre Länge schwankt zwischen 18 und $28 \mathrm{~mm}$. und die Breite zwischen 9 und $14 \mathrm{~mm}$. Die beiden Enden des Blattes sind rundig oder wenig vertieft. Die Seitennerven sind jederseits 6 vorhanden. Der Blattstiel ist etwa $2 \mathrm{~mm}$.

Diese Blätter gehören unzweifelhaft zu Leguminosae durch die morphologischen Eigenschaften, und stimmen wahrscheinlich mit der in Formosa lebenden Art Entada formosana KANEHIRA überein.

\section{Euphorbiaceae}

Sapium sebiferum RoxB. var. pleistoceaca MIKI

Tafel VII, Fig. 8 a, b c, d.

Sapium sebiferum Roхв. var.: Mткі (1933) S. 624, Taf. K, Fig. 4 T-V; (1937) S. 320 , Taf. 8 E, Fig. 7 D; (1938) S. 220, Fig. 5 I.

Die zahlreichen kleinen Samen wurden in der Tuffitschicht bei Kazusa gefunden. 


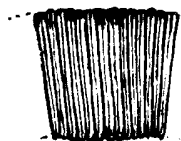

Textfig. 2. Der Schnitt der

Samenschale. vergrössert.

Die betreffenden Fossilien sind etwa $5 \mathrm{~mm}$. lang und etwa $5 \mathrm{~mm}$. breit. Ihre Samenschale zeigt die Sammlung der palisadenartig nebeneinanderstandenen Sklerenchmzellen.

Diese kleinen Samenreste stimmen unzweifelhaft mit der von MrkI als die Varietät der lebenden Art beschriebenen Pflanze Sapium sebiferum RoxB. var. pleistoceaca MiKI überein.

\section{Buxaceae \\ Buxus japonica MUELL \\ Tafel VII, Fig. 13 a-g.}

Buxus japonica MueLn: Мıк (1937) S. 320, Fig. 7 A, B; (1941) S. 281, Fig. 16 D.

Ich fand diese zahlreichen Blattreste in den Schichten bei Kutinotsu und Kazusa.

Die etwa länglich-ovalförmigen, an der Spitze etwas vertieften, kurzgestielten, lederartigen, fiedernervigen Blätter sind 19-30 mm. lang und 7-12 mm. breit. Die Seitennerven gabeln einigemal und erreichen den Rand.

Die vorliegenden Reste stimmen vollkommen mit der heutigen lebenden Art Buxus japonica MuELl überein.

\section{Aceraceae \\ Acer pictum ThunB. \\ Tafel VII, Fig. 3, 4 a, b.}

Acer pictum Thunb.: Nath. (1883) S. 247, Taf. 12, Fig. 2-8; (1888) S. 38, Taf. 13, Fig. 1, 2; Endo (1934) S. 247, Taf. 29, Fig. 3, 4, 7, 8, 9; Taf. 30, Fig. 2; Taf. 31, Fig. 4, 5; Taf. 32, Fig. 3, 6 .

Die vorliegenden Ahornblätter, die die unvollständigen Fragmente sind, haben fünf strahlige Leitbündel, und sind ganzrandig.

Sie stimmen unzweifelhaft mit der gegenwartig lebenden Art Acer pictum Thuns. überein.

Acer palmatum ThunB.

Tafel VII, Fig. 5, 6, 7 a, b.

Acer palmatum Thunb.: Miki (1941) S. 283, Fig. 17 B-Cd. 
Einige Ahornblätter, die unvollständig sind, sind fast rundförmog, am Rande gezähnt, und haben funf oder sieben strahligen Leitbündel. Ihre Basis ist etwas herzförmig.

Diese betreffenden Blätter gehören unzweifelhaft zu Acer palmatum Thunb, durch die morphologischen Merkmale.

\section{Acer cf. parviflorum F. et S. (?)}

Tafel VII, Fig. 1 a, b.

Zwei Ahornfrüchte wurden in den Schichten bei Kazusa und Hasudake gefunden. Sie gehören wahrscheinlich zu Acer parviflorum F. et S.

\section{Acer cf. diabolicum BL. (?) \\ Tafel VII, Fig. 2.}

Acer diabolicum Bu.: Endo (1934) S. 249, Taf. 31, Fig. 1, 2; Taf. 34, Fig. 2 ; Taf. 35, Fig. 4, 5, 15, 18.

Die vorliegende Ahornfrucht wurde in der Tuffitschicht bei Kazusa gefunden.

Diese Frucht ist kleiner als die von Acer diabolicum BL., aber gehört wahrscheinlich zu Acer diabolicum BL. durch die morphologischen Merkmale.

\section{Sapindaceae}

Xanthoceras cf. sorbifolia BGE.

Tafel VII, Fig. 10.

Xanthoceras sorbifolia BGE.: Miki (1937) S. 322, Taf. 9 E, Fig. 5 I, 10 C, D.

Ich fand das kleine Blatt in der Tuffitschicht bei Kazusa.

Das vorliegende Blatt ist etwas lang-elliptisch, am Rande gezähnt, 25 $\mathrm{mm}$. lang und $11 \mathrm{~mm}$. breit. Es scheint, dass das betreffende Blatt das terminale ist. Die Sekundärnerven verlaufen camptodromig.

Dieses Blatt stimmt wahrscheinlich mit der in China und Mandschurei lebenden Art Xanthoceras sorbifolia BGE. überein.

\section{Rhamnaceae}

\section{Paliurus nipponicus MikI}

Tafel VII, Fig. 11 a, b, c, d.

?Zizyphus tiliaefolius UN(..: Nath (1888) S. 208, Taf. 4, Fig. 5.

Paliurus nipponicus Miкт: Mтк (1933) S. 624, Taf. Q-U, Fig. 2 F-J ; (1937) S. 324, Taf. 9 H, I, Fig. 8 A-E; (1938 a) S. 216, Fig. 2 A; S. 220, Fig. 5 E; S. 224, Fig. 6 A; (1941) S. 285, Fig. 17 G.

Diese etwa vollständig erhaltenen Früchte wurden in der Tuffitschicht bei Kazusa gefunden.

Die vorliegende Frucht wird von dem verbreiteten Flügel etwas rundlich umgeben, dessen Breite zwischen 8 und $16 \mathrm{~mm}$. schwankt, und hat den von dem Flügel umgebenen Fruchtknoten. 
Sie stimmt vollkommen der von MiKI beschriebenen Art Paliurus nipponicus MıKI überein.

\section{Tiliaceae}

Tilia cf. japonica Simk.

Tafel VII, Fig. 9.

Tilia cf. cordata MiLt.: Nath. (1888) S. 34, Taf. 10, Fig. 11.

T. japonica Simk.: ENdo (1940) S. 68, Taf. 4, Fig. 1; Taf. 8, Fig. 19 ; Miki (1938 a) S. 229, Taf. 4 H, Fig. $10 \mathrm{~K}$; (1941) S. 286 , Fig. 18 B, C.

Das abgebildete Stück stellt ein Fragment eines Schirmblattes dar, dessen Form und Grösse lassen es zu Tilia japonica Simk. wahrscheinlich gehören.

\section{Theaceae}

Camellia japonica L.

Tafel VIII. Fig. 2 a-c.

Camellia japonica L.: M M $(1938$ a) S. 220, Fig. 5 K; (1941) S. 288, Fig. 18 D.

Einige Blätter sind etwas elliptisch, am Rande fein gezähnt, lederartig und etwa $40 \mathrm{~mm}$. breit.

Die Sekundärnerven, die jederseits 7 vorhanden sind, verlaufen campto dromartig.

Die vorliegenden Blätter stimmen vollkommen mit der lebenden Art Camellia japonica L. überein

Stewartia cf. monadelpha S. et $Z$.

Tafel VII, Fig. 12.

Stuartia monadelpha S. et Z.: NATH. (1883) S. 66, Taf. 14, Fig. 11, 12.

S. monadelpha FLORIN : Kon'No (1931) S. 145, Taf. 14, Fig. 6.

S. $\quad$ monadelpha S. et Z.: Miki (1937) S. 324, Fig. $8 \mathrm{~K}$.

Stewartia monadelpha S. et Z.: Mrki (1941) S. 288, Fig. 18 E, G.

Ich fand das unvollständig erhaltene Blatt in der Tuffitschicht bei Kazusa.

Dieses Blatt ist verkehrt-eiförmig, am Rande fein gezähnt, und etwa $23 \mathrm{~mm}$. breit.

Der betreffende Rest stimmt wahrscheinlich mit der lebenden Art Stewartia monadelpha S. et $Z$. überein.

\section{Liliaceae}

Smilax China L.

Tafel VIII, Fig. $1 \mathrm{a}-\mathrm{m}$.

Die zahlreichen kleinen Blätter wurden von mir in der Tuffitschicht bei Kazusa gefunden. 
Die vorliegenden Reste sind kreisförmig, oder auch etwas länger oder breiter kreisförmig, an der Spitze etwas vertieft oder etwas konvex, und an der Basis rierenförmig oder kreisförmig.

Ihre Länge schwankt $z$ wischen 13 und $29 \mathrm{~mm}$. und die Breite zwischen 12 und $34 \mathrm{~mm}$. Das seitliche Hauptnervenpaar lauft den Zwischenraum des Hauptmittelnervs und Randes von der Basis aus zu der Spitze und das unterste fein Seitennervenpaar entlang des Randes aufwärts.

Die jetzt in Japan lebenden Blätter haben das seitliche Hauptnervenpaar oder zwei seitliche Hauptnervenpaare. Ihre kleinen Formen zeigen gewöhnlich das erstere und die grossen Formen meistens die letztere.

\section{Ebenaceae}

\section{Diospyros Kaki Thunb. \\ Tafel VIII, Fig. 3.}

Das vorliegende grosse Blatt ist etwas elliptisch, ganzrandig, lederartig, etwa $12 \mathrm{~cm}$. lang und $8.2 \mathrm{~cm}$. breit. Seine Spitze ist kurzig gespitzt und seine Basis etwas keilförmig. Die Sekundärnerven, die jederseits 7 vorhanden sind, verlaufen camptodromig.

Dieses Blatt gehört unzweifelhaft zu Diospyros Kaki Thuns.

\section{Geologische Altersbestimmung und Klimatische Verhältnisse}

Um das geologische Alter der pflanzenführenden Ablagerungen festzustellen, müssen die verschiedenen Pflanzenfossilien gesammelt werden, dann wird jedes einzelne Fossil morphologisch untersucht, um die Charakteristik der Fossilienflora festzulegen. Häufig findet man Pflanzenreste junger Ablagerungen, deren Arten heute in Japan ausgestorben sind. Je häufiger diese Arten in den Pflanzenfossilien vorkommen, desto ïlter ist das geologische Alter, jünger ist es, je weniger diese Arten darin enthalten sind. Es hat stratigraphisch grossen Wert, dass sich die Oya-Formation auf die Alttertiärschichten diskordanzig aufhäuft.

Überblicken über Flora, die in sechs verschiedenen Lokalitäten vorkommen.

1) Oshima. Hier kommen Fagus crenata BL., Carpinus carpinoides Makino, Cinnamomum sp. vor.

2) Kazusa. Von dieser Lokalität habe ich Metasequoia cf. japonica Mikı, Glyptostrobus pensilis KocH, Thuja Standishii CARr., Carex (?) sp., Carpinus carpinoides MaKino, C. Tschonoskii Max., Fagus crenata Bl., $F$. cf. ferruginea AIT.(?), Magnolia cf. Kobus DC., Quercus stenophylla MAKINo, Zelkova Ungeri Kovats, Celtis sp., Wistaria cf. brachybotis S. et Z.(?), Entada formosana KANEHIRA, Buxus japonica MuelL, Rosa multiflora Thunb., Camellia japonica L., Stewartia cf. monadelpha S. et Z., Sapium 
sebiferum Rox. var., Paliurus nipponicus Mıк, Liquidambar cf. formosana HANCE, Xanthoceras cf. sorbifolia BGE., Smilax China L., Acer pictum THunB., A. cf. diabolicum BL.(?), A. cf. parviflorum F. et S. (?), Populus Inouei n. sp., Salix cf. amygdalina L. var., Salix sp. (a), S. sp. (b), S. sp. (c), gesehen.

3) Hasudake. Hier kommen Quercus cf. gilva BL., Buxus japonica Muell, Acer cf. parviflorum F. et S. (?) vor.

4) Kashiyama. Die Pflanzenreste, die hier vorkommen, scheinen zu folgenden Arten zu gehören: Dryopteris taiwanensis Christ., Pinus aff. Thunbergii PArl., Carex (?) sp., Phyllostachys (?) sp., Carpinus carpinoides MaKino, Corylus cf. ligniatus MiKI, Fagus crenata BL., Quercus sp., Zelkova Ungeri Kovats, Buxus japonica Mueld, Rosa sp., Camellia japonica L., Smilax China L., Tilia cf. japonica Simk., Acer pictum ThunB., A. palmatum ThunB., Cinnamomum cf. Camphora SieB.

5) Ôtomari. Hier kommen Carpinus carpinoides Makino, C. Tschonoskii Max., C. sp., Fagus crenata Bl. Celtis sp., Buxus japonica Muell, Liquidambar cf. formosana HANCE, Acer palmatum THunB., vor.

6) Ô. Quercus sp.

Versuchen wir jetzt das geologische Alter der Ôya-Formation, welche die oben erwähnten zahlreichen Fossilien geliefert hat, festzustellen.

Ôya-Formation hat Dryopteris taiwanensis CHRIST., Metasequoia cf. japonica MIKI, Glyptostrobus pensilis KocH, Pinus aff. Thunbergii PARL., Carpinus sp., Corylus cf. ligniatus MiKI, Fagus cf. ferruginea AIT., Zelkova Ungeri Kovats, Entada formosana KANEHIRA, Sabium sebiferum RoxB. var., Paliurus nipponicus Mrki, Liquidambar cf. formosana HANCE, Xanthoceras cf. sorbifolia BGE., Populus Inouei n. sp., welche die schon in Japan ausgestorbenen Pflanzen sind, geliefert.

Nach der Beschreibung Miki's kann man Metasequoia, Glyptostrobs, und Liquidambar bis zur Pliozänzeit erkennen, aber nicht bis zur Quartärzeit. Und auch Sapium sebiferum RoxB. var. und Paliurus nipponicus MikI sind von MikI aus den Oberpliozän- und Unterpleistozänablagerungen bei Akashi und Kyoto beschrieben worden. Daher gehört die Ôya-Formation der Tertiärzeit an. Die zwölf Arten der Akashi- und Ôya-Flora sind identisch: Thuja Standishii CARr., Carex sp., Fagus crenata Bl., Zelkova Ungeri Kovats, Magnolia Kobus DC., Buxus japonica Mueld, Rosa multiflora Thunb., Stewartia monadelpha S. et Z., Sapium sebiferum Roxв. var., Paliurus nipponicus MIKI, Xanthoceras sorbifolia BGE., Salix amygdalina L. var.(?). Daher hat Oya-Flora die Ähnlichkeit mit der Akashi-Flora oder auch mit der Mogi-Flora (?).

NAThozst hat einmal geäussert, dass die Flora von Mogi entweder dem Ende der Tertiärzeit oder dem Beginn der Quartärzeit angehöre, sie sei, aber nicht vorpliozän. Mrki hat später die Meinung ausgesprochen, dass Akashi-Flora mit der Mogi-Flora gleichalterig sei, nämlich Oberpliozän. 
Aus den oben erwähnten Tatsachen könnte das geologische Alter der ÔyaFlora in die Oberpliozänzeit fallen.

\begin{tabular}{l|c|c|c}
\hline & \multicolumn{2}{|c|}{ Oberpliozăn } & Unterpleistozän \\
\cline { 2 - 4 } & $\begin{array}{c}\text { Akashi-Flora } \\
\text { (nach MIKI) }\end{array}$ & Ôya-Flora & $\begin{array}{c}\text { Yamashiro-Flora } \\
\text { (nach MIkI) }\end{array}$ \\
\hline Ausgestorbene Arten & $18(31 \%)$ & $14(32 \%)$ & $6(26 \%)$ \\
\hline Pflanzen mit den!Dörnern & $11(19 \%)$ & $4(9 \%)$ & $2(9 \%)$ \\
\hline Koniferen & $6(10 \%)$ & $4(9 \%)$ & $1(4 \%)$ \\
\hline Wasser-oder Marschpflanzen & $4(7 \%)$ & $4(9 \%)$ & $7(30 \%)$ \\
\hline
\end{tabular}

Die vergleichende Übersicht jeder Flora.

Vierzehn der Ôya-Flora angehörige Arten sind in Japan ausgestorben (32\%). Diese Prozentzahl scheint das geologische Alter der jüngeren Ablagerungen $\mathrm{zu}$ bestimmen.

Paliurus nipponicus MIKI und Sapium sebiferum RoxB. var. sind die in der Meeresküste lebenden Pflanzen, und ihre am nächsten verwandten Arten verbreiten in China, Mandchurei, Formosa und Liukiuinseln. Es ist sehr interessant, dass sie in der Ôya-Formation von Kiushiu gefunden worden sind.

In den in der Ôya-Formation gefundenen Pflanzenfossilien sind die Arten, die besonders von der Temperatur abhängig waren, Thuja Standishii CARr., Fagus crenata BL., Buxus japonica Muell, usw. In Südjapan wachsen sie wild $z$ wischen 800 und 1000 Meter über dem Meeresspiegel. Nach den Umständen könnte das Klima der Ôya-Zeit kälter $4-5^{\circ} \mathrm{C}$ als gegenwärtig gewesen sein.

\section{Zussammenfassung}

1) Vierzehn der Ôya-Flora angehörige Arten sind in Japan ausgestorben und die Ähnlichkeiten der Ôya- und Akashi-Flora sind identisch.

2) Ôya-Flora ist identisch mit der Akashi-Flora und Mogi-Flora (?).

3) Das geologische Alter der Ôya-Formation könnte in die Oberpliozänzeit fallen.

4) Ôya-Flora enthält die mit China, Mandchurei, Formosa und Liukiuinseln zusammenhängenden Arten: Dryopteris taiwanensis CHRIST., Metasequoia japonica MıкI, Glyptostrobus pensilis Косн, Carpinus Tschonoskii MAX., Zelkova Ungeri KovATS, Entada formosana KANEHIRA, Buxus japonica 
Muell, Camellia japonica L., Sapium sebiferum Roxв. var., Paliurus nipponicus MIKI, Liquidambar formosana HANCE, Xanthoceras sorbifolia BGE., Smilax China L. Unter ihnen befinden sich die in Japan lebenden Arten Carpinus Tschonoskii MAX., Camellia japonica L., Buxus japonica MuELL. und Smilax China L.

5) Das Klima aus der Ôya-Flora könnte $4-5^{\circ} \mathrm{C}$ kälter als das heutige Klima sein.

\section{Literatur}

Chaney, R. W. (1940): Tertiary Forests and Continental History. Bull. Geol. Soc. Amer. 51, 469-488

Endo, S. (1928): A new Palaeogene species of Sequoia. Jap. Jour. Geol. and Geogr. $6,27-29$.

Ders. (1931): Cenozoic fossil plants. (jap.)

Ders. (1933) : Cenozoic fossil plants, supplement. (jap.)

Ders. (1934): Some Japanese Cenozoic Plants. I. On the Fossil Acer from the Siobara Pleistocene Plant Beds: Jap. Jour. Geol. and Geogr. 11, 239-253.

Ders. (1936): New fossil species of Sequoia from the Far-East. Proc. Imp. Acad. Tokyo $12,172-\mathrm{I} 75$.

Ders. (1938 a): Über die Fossilien von Taxodiaceae. (jap.) Jour. Geol, Soc. Japan $45,468-470$.

Ders. (1938 b): On fossil plants from the environs of Sendai. (jap.) Jour. Geol. Soc. Japan 45, 618-620.

Ders. (1939 a): A pleistocene Flora from Kagoshima, Kyushu, Japan. Jour. Geol. Soc. Japan 46, 204-208.

Ders (1939 b): Some new and interesting Miocene plants from Tyosen (Korea). Jubilee Pub. Comm. Prof. Yabe 333-349.

Dres. (1940): A Pleistocene Flora from Siobara, Japan. Sci. Rep. Tohoku Imp. Univ. Sendai, 2nd ser. 21, No. 1.

Ders. (1950 a): On the Fossil Acer from Japan, Korea, and South Manchuria. Short Papers from the Inst. of Geol. and Paleont. Tohoku Univ. Sendai. No. 1, 11-16.

Ders. (1950 b) : On the Fossil Carpinus from Japan and Korea. Short Papers Geol. and Paleont. Tohoku Univ. Sendai No. 2, 51-57.

Ders. (1951): On the Fossil Acer from Japan, Korea and South Manchuria. Short Pap. Geol. and Paleont. Tohoku Univ. Sendai. No. 3, 52-58.

Endo S. and Morita, H. (1932): Notes on the genera Comtoniphyllum and Liquidam. bar. Sci. Rep. Tohoku Imp. Univ. Sendai Japan 2nd Ser. 15, 47-53.

Endo, S. and Okuтsu, H. (1936): Glyptostrobus cone from the Liriodendron Bed near Sendai. Proc. Imp. Acad. Tokyo 12, 138-140.

ENGLER-Gila (1924): Syllabus der Pflanzenfamilien.

Fuzroka, K. (1951): Notes on some Tertiary Plants from Tyosen (Korea). II. Trans. Proc. Palaeont. Soc. Japan N.S., No. 3, 67-74.

HeER, O. (1869) : Flora Fossilis Alaskana. Kongl. Svenska Vetenskaps-Akad. Handl. B. 8 No. 4 . 
Hu, H. H. and Chaney, R, W. (1940): A Miocenc Flora from Shantung Province, China. Carnegie Inst. Publ., 507.

KANEhiRA, R. (1936): Formosan Trees. (jap.)

Kirchheimer, F. (1939): Tertiäre Dikotyledonenreste und ihr systematischer Wert. Flora N. F. B. 33, 239-296.

Ders. (1939) : Über die botanische Zugehörigkeit weiterer Frucht-und Samenreste, besonders aus den Braunkohlenschichten Sachsens. Planta B. 29, 262-278.

KolwaI, K. (1915): Notes on a Plant Bed exposed at Sanjunin-Machi in Sendai. Sci. Rep. Tohoku Imp. Univ., (II)

Kon'so, E. (1931) : Känozoische fossile Flora in Mittelshinano. (jap.) Geol. in Mittelshinano von $\mathrm{F}$. Honma $91-156$.

Kryshtofovich, A. (1920): A New fossil palm and some flora of Japan. Jour. Geol. Soc. Tokyo 27, 1-20.

Makino, T. u. Nemoto, K. (1931): Flora von Japan. (jap.)

Miki, S. (1933): On the Pleistocene flora in Prov. Yamashiro with the description of 3 new species and 1 new variety. Bot. Mag. Tokyo 47, 619-631.

Ders. (1937): Plant fossils from the Stegodon Beds and Elephas Beds near Akashi. Jap. Jour. Bot. 8, 303-341.

Ders. (1938 a): On the change of Japan since the Upper Pliocene and the floral composition at the present. Jap. Jour. Bot. 9, 213-251.

Ders. (1938 b) : Über die Reste von Wasserpflanzen Asiens. (jap.). Jap. Jour. Limnology Tokyo 8, 410-416.

Ders. (1939): On the remains of Pinus trifolia n. sp. in the Upper Tertiary from Central Honsyu in Japan. Bot. Mag. Tokyo 53, 239-246.

Ders. (1941): Floral remains of the Conifer Age at Manzidani Near Nishinomiya, Japan (Preliminary note). Jap. Jour. Bot. 11, 377-383.

Ders. (1950): Taxodiaceae of Japan, with Special Reference to its Remains. Jour. Inst. Polyt. Osaka City Univ. Ser. D, Biol., 1, 63-77.

Ders. (1953) : Metasequoia. (jap.)

Morita, H. (1931): On New Species of the Genera Cinnamomum and Smilax from the Miocene Deposits of Oguni-machi, Uzen Province, Japan. Jap. Jour. Geol. and Geogr. 9, 1-8.

Nathorst, A. G. (1883); La flore fossile du Japan. Kgl. sv. Vet Akad. Handl. 20, 3-92.

Ders. (1888); Zur fossilen Flora Japan. Pal. Abh. 4.

Ogata, M. (1928-1940): Icones Filicum Japoniae. vol. 1-8.

OrsHI, S. (1950): Illustrated Calalogue of East-Asiatic Fossil Plants. (jap.)

Oor, J. (1953) : Flora of Japan. (jap.)

Ротоní́ u. Gothan (1921): Lehrbuch der Paläobotanik.

Shimakura M. (1939): The past and origin of Coniferous plants in Japan. Jub. Publ. Prof. Yabe 233-253.

Shiкama, T. (1938): On the Kobe Group and its flora. Jour. Geol. Soc. Japan 45, 621-640.

Ders. (1952): Die Quartärzeit. (jap.)

Tanal, T. (1952): Notes apropos de quelques plantes fossiles dans le Groupe d'Ennichi. Tran. and Proc. Paleont. Soc. Jap. N. ser. No. 8, 231-236.

YABE, H. (1929): The Latest Land Connection of the Japanese Islands to the Asiatic Continent. Proc. Imp. Acad. 5, 167-169.

Ders. (1929): Geological Age of the Latest Continental Stage of the Japanese Islands. Proc. Imp. Acad. 5, 430-433.

YaBE, H. u. Endo, S. (1930): Über die fossilen Flora und die Geologie von Mogi, Prov. Hizen, Japan. (jap.) Jour. Geogr. 42, 599-607.

ZiтTes, K. A. (1890): Handbuch der Paläontologie. 
K. TAKahashi

\section{Zur fossilen Flora aus der Ôya-Formation} von Kiushiu, Japan

\section{Tafelerklärungen}




\section{Erklärung der Tafel I}

Fig. 1. Dryopteris taiwanensis CHRIsT.

Fig. 2a-b. Metasequoia cf. japonica MIKI

Fig. 3. Thuja Standishii CARR.

Fig. 4. Glyptostrobus pensilis KoOH

Fig. 5a-f. Pinus aff. Thunbergii PARI.

Fig. 6 a, b. Carex (?) sp.

Fig. 7 a, b, c. Phyllostachys (?) sp.

Fig. 8. Salix cf. amygdalina var. (?)

Fig. 9 a, b, c. Salix sp. (a)

Fig. 10 a, b. Salix sp. (b)

Fig. 11. Salix sp. (c)

Fig. 12 a, b. Populus Inouei n. sp.

Die Objekte sind in natürlicher Grösse gezeichnet. 


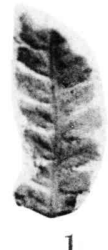

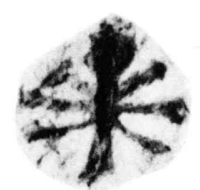

a

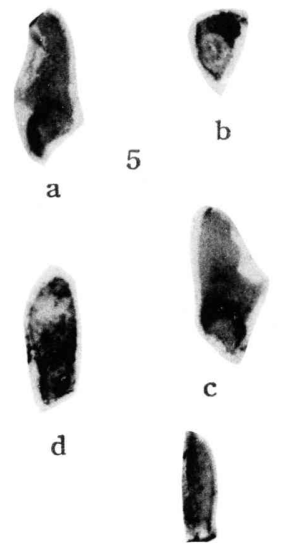

e

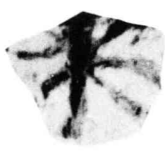

b

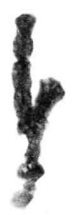

3

8
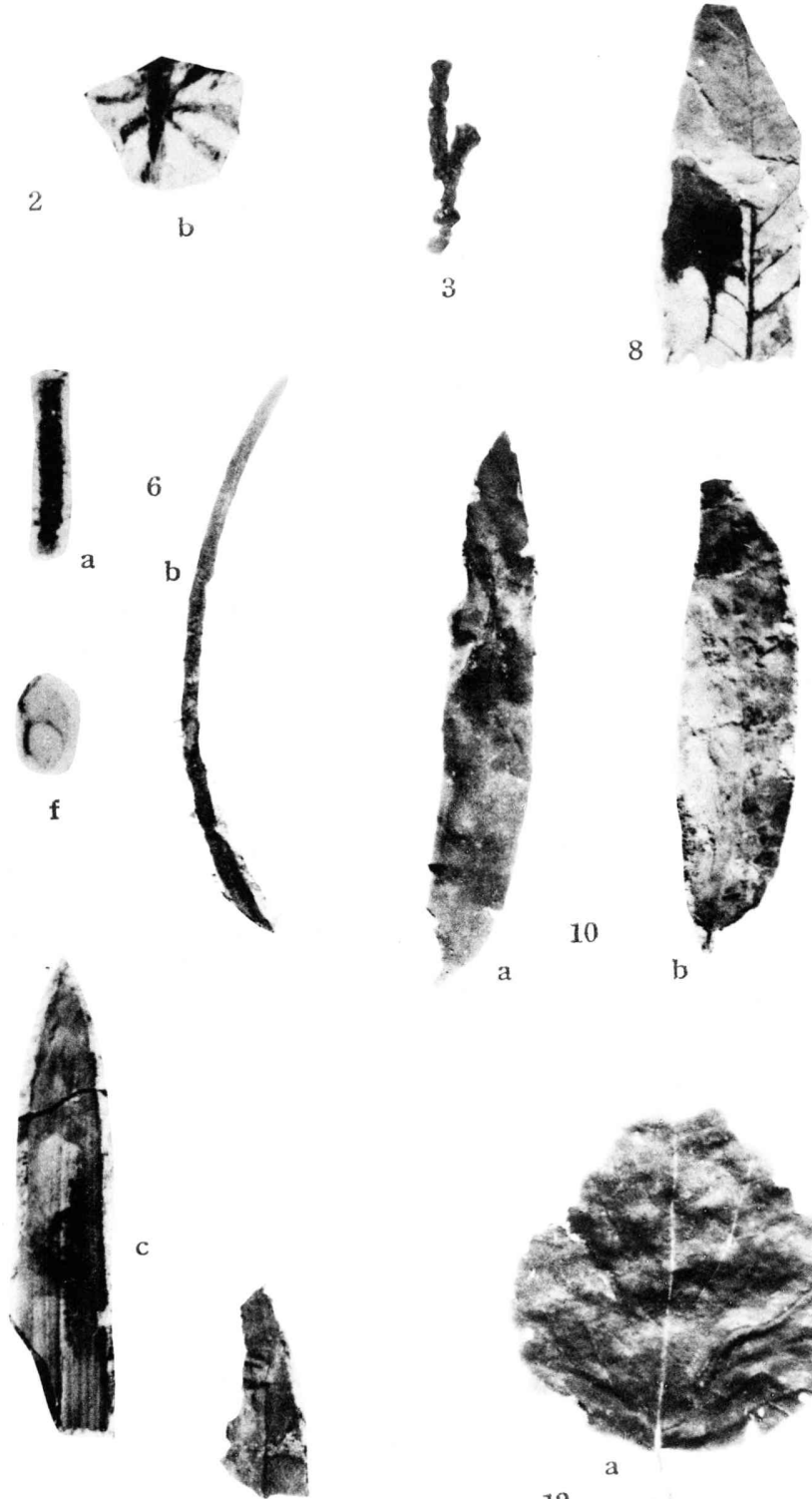

C

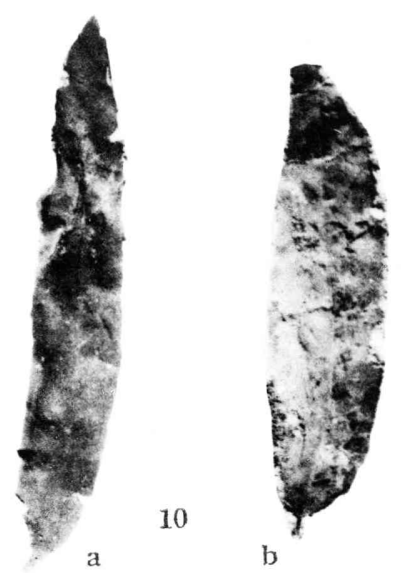

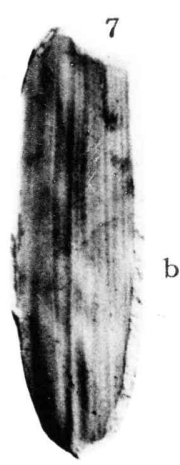
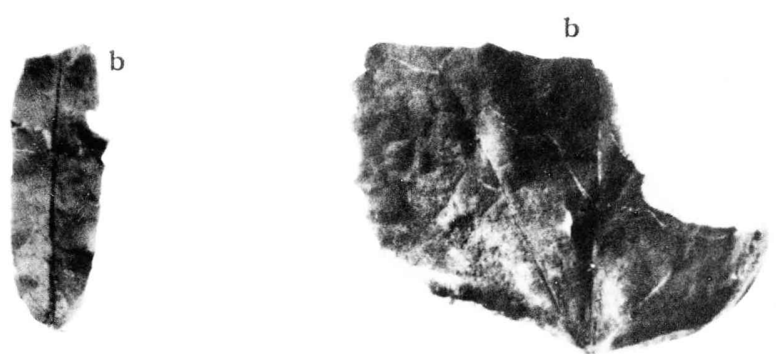


\section{Erklärung der Tafel II}

Fig. 1-10. Carpinus carpinoides Makino (?)

1-8: Blätter ; 9, 10: Fruchtbecher.

Fig. 11. Carpinus Tschonoskii MAX.

Fruchtbecher.

Fig. 12. Carpinus sp.

Fruchtbecher.

Die Objekte sind in natürlicher Grösse gezeichnet. 

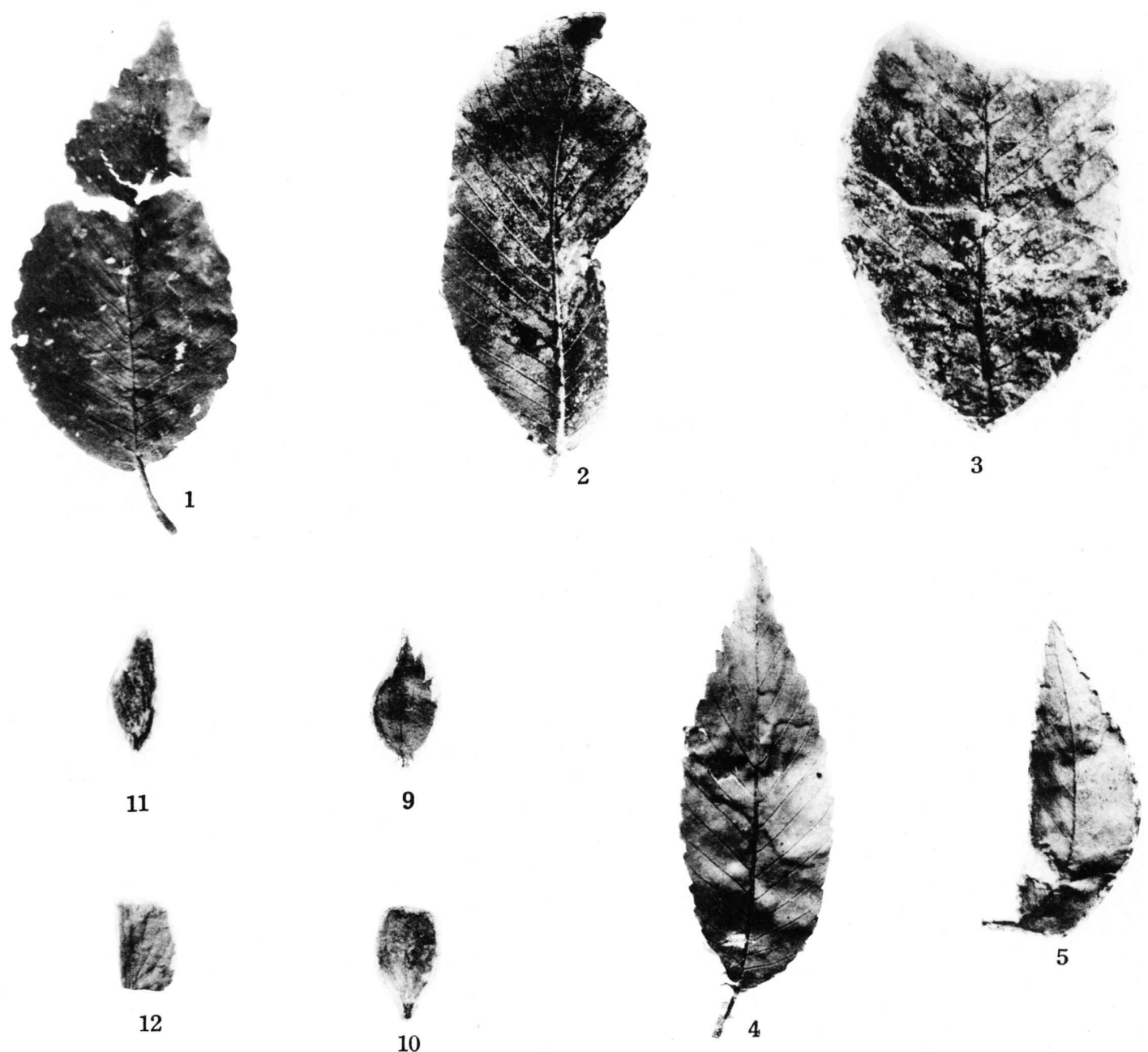

12

10
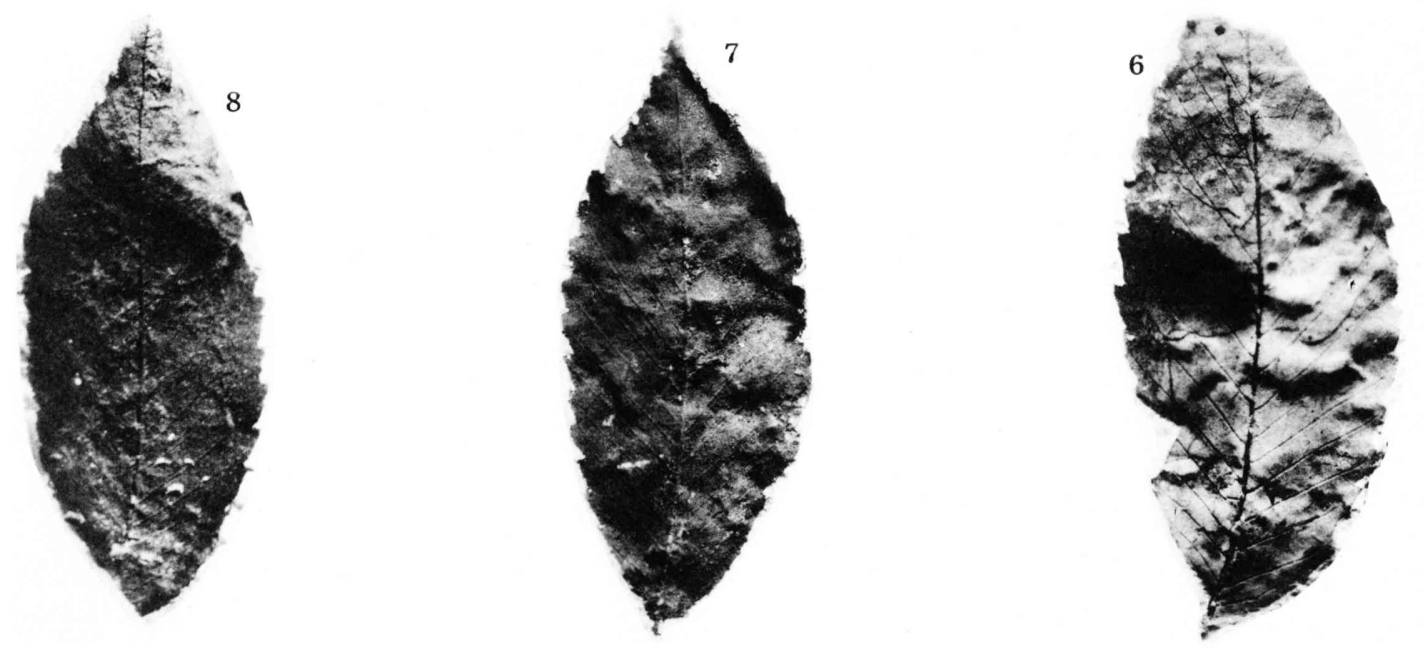


\section{Erklärung der Tafel III}

Fig. 1-5. Carpinus carpinoides Marino

Fig. 6. Carpinus Tschonoskii Max. (?)

Fig. 7-12. Fagus crenata BL.

Die Objekte sind in natürlicher Grösse gezeichnet. 

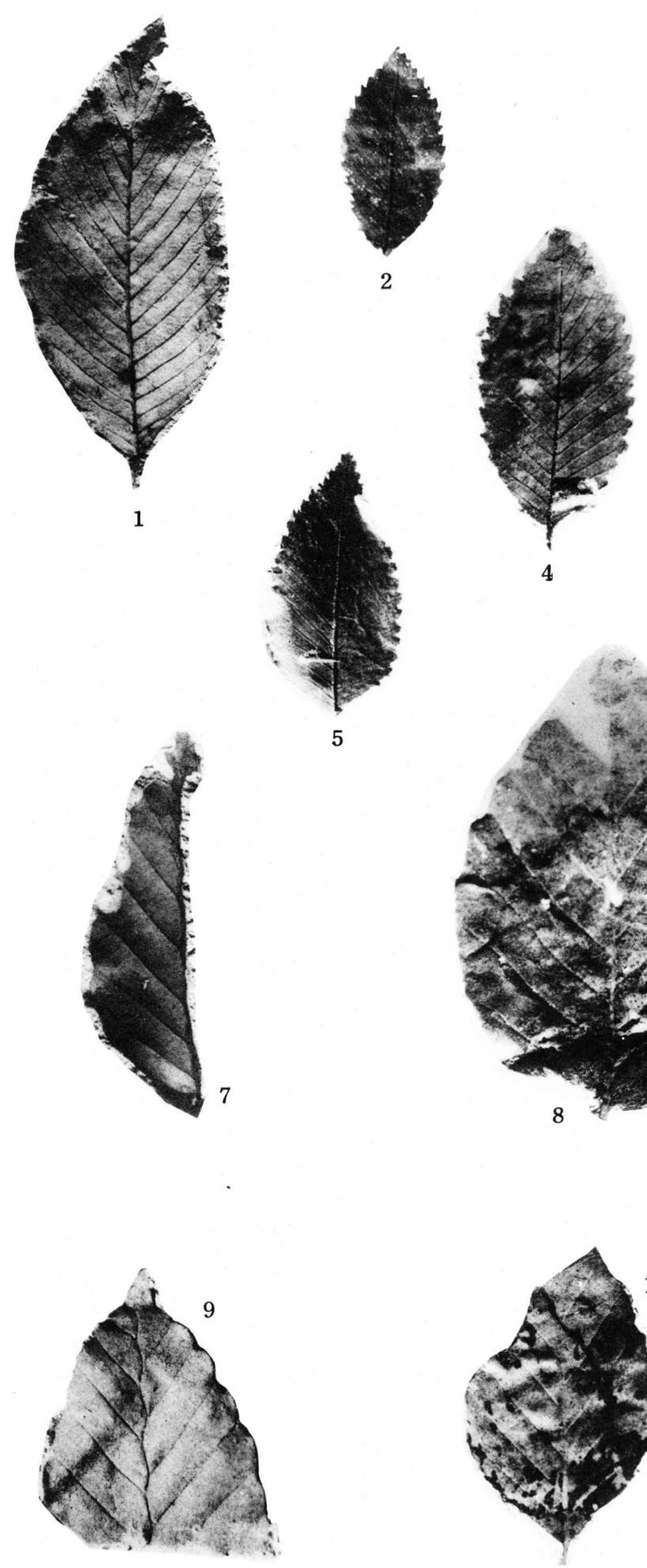
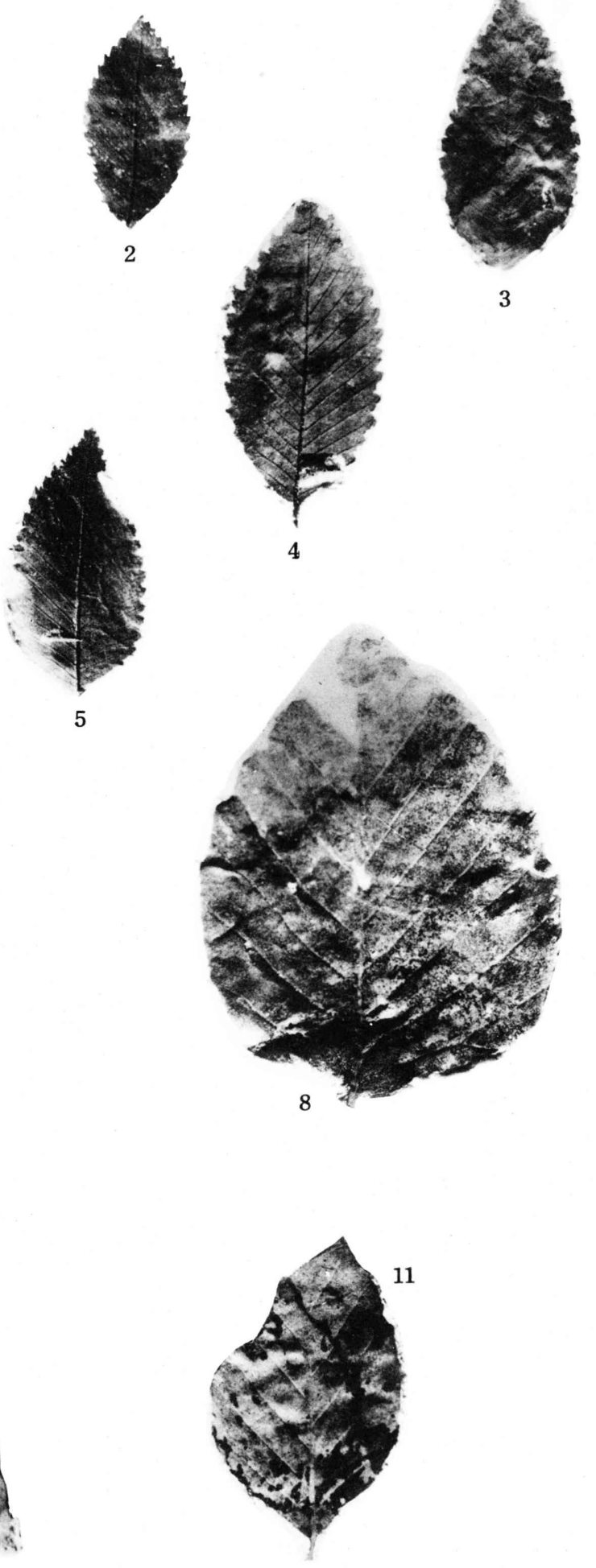
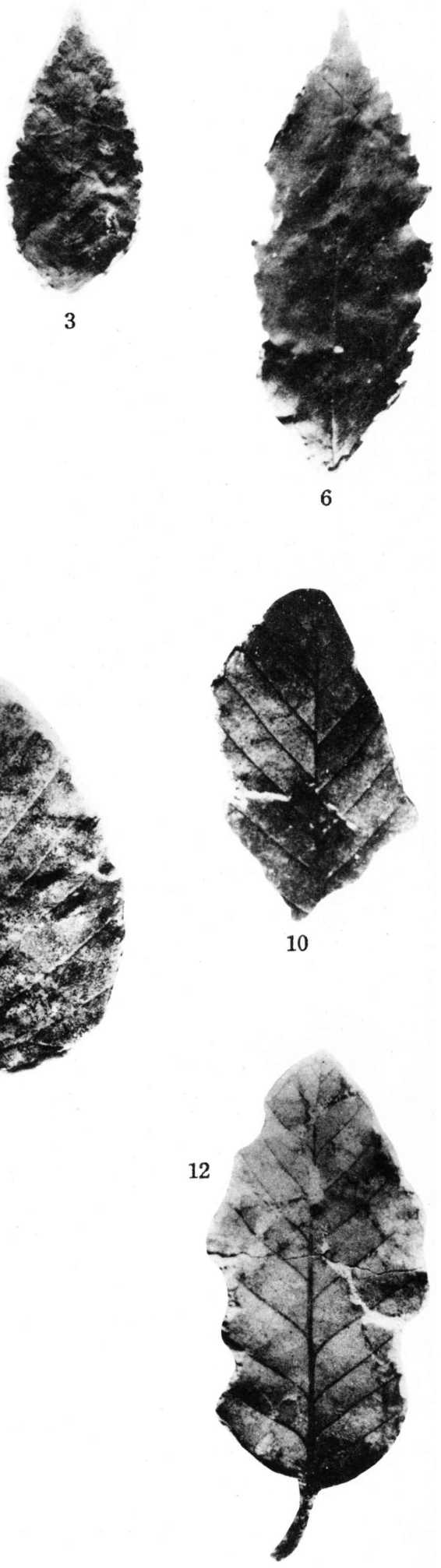


\section{Erklärung der Tafel IV}

Fig. 1-8. Fagus crenata BL.

Fig. 9 a, b. Corylus cf. ligniata MikI

Die Objekte sind in natürlicher Grösse gezeichnet. 
Mem., Fac. Sci., Kyushu Univ., Ser. D, Vol. V, No. 1
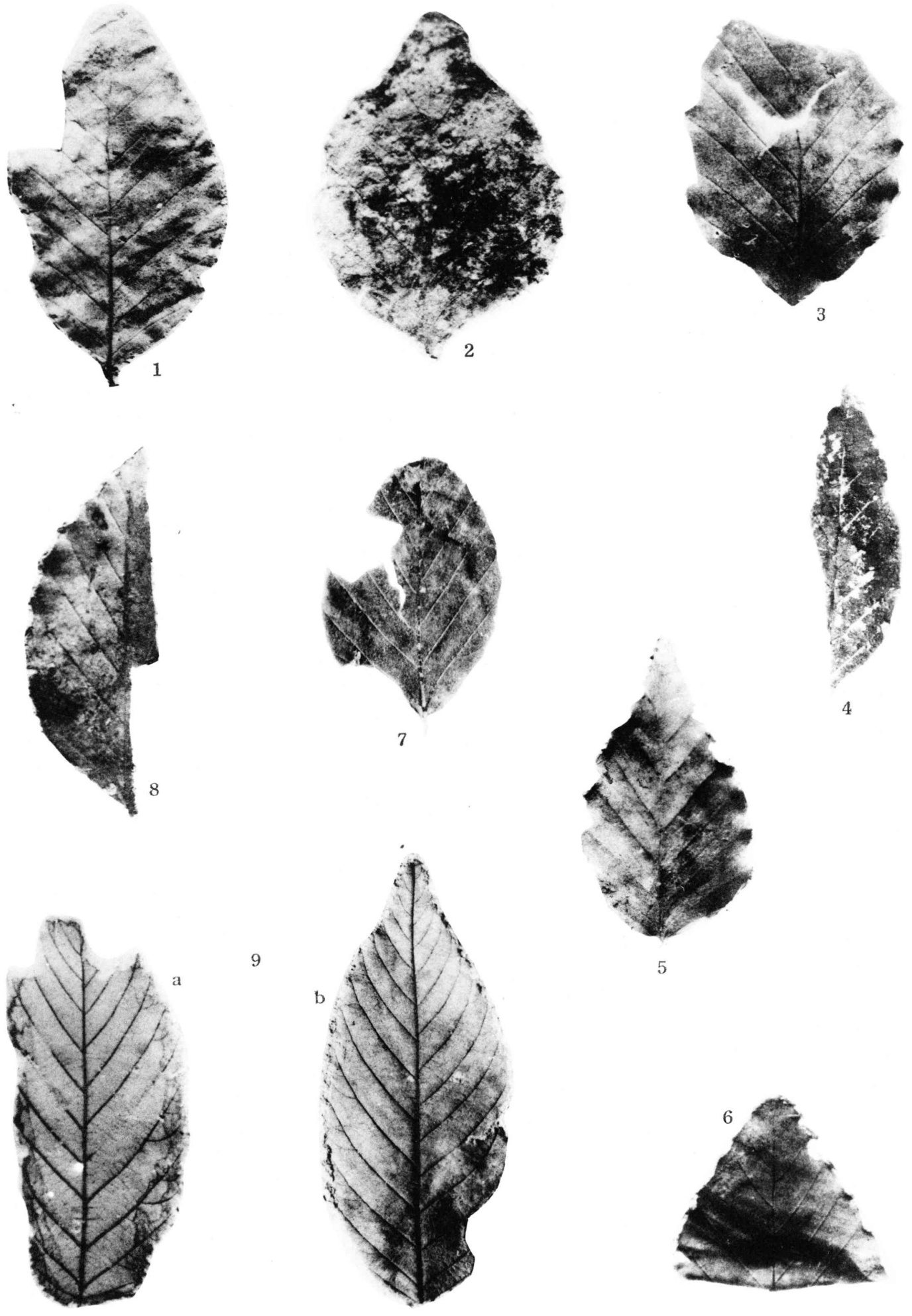


\section{Erklärung der Tafel V}

Fig. 1. Fagus cf. ferruginea Ait. (?)

Fig. 2-4. Quercus sp.

Fig. 5. Quercus cf. gilva BL.

Fig. 6. Quercus stenophylla Makino

Fig. 7. Celtis sp.

Fig. 8 a-g. Zelkova Ungeri Kovats

Fig. 9. Magnolia cf. Kobus DC.

Die Objekte sind in natürlicher Grösse gezeichnet. 

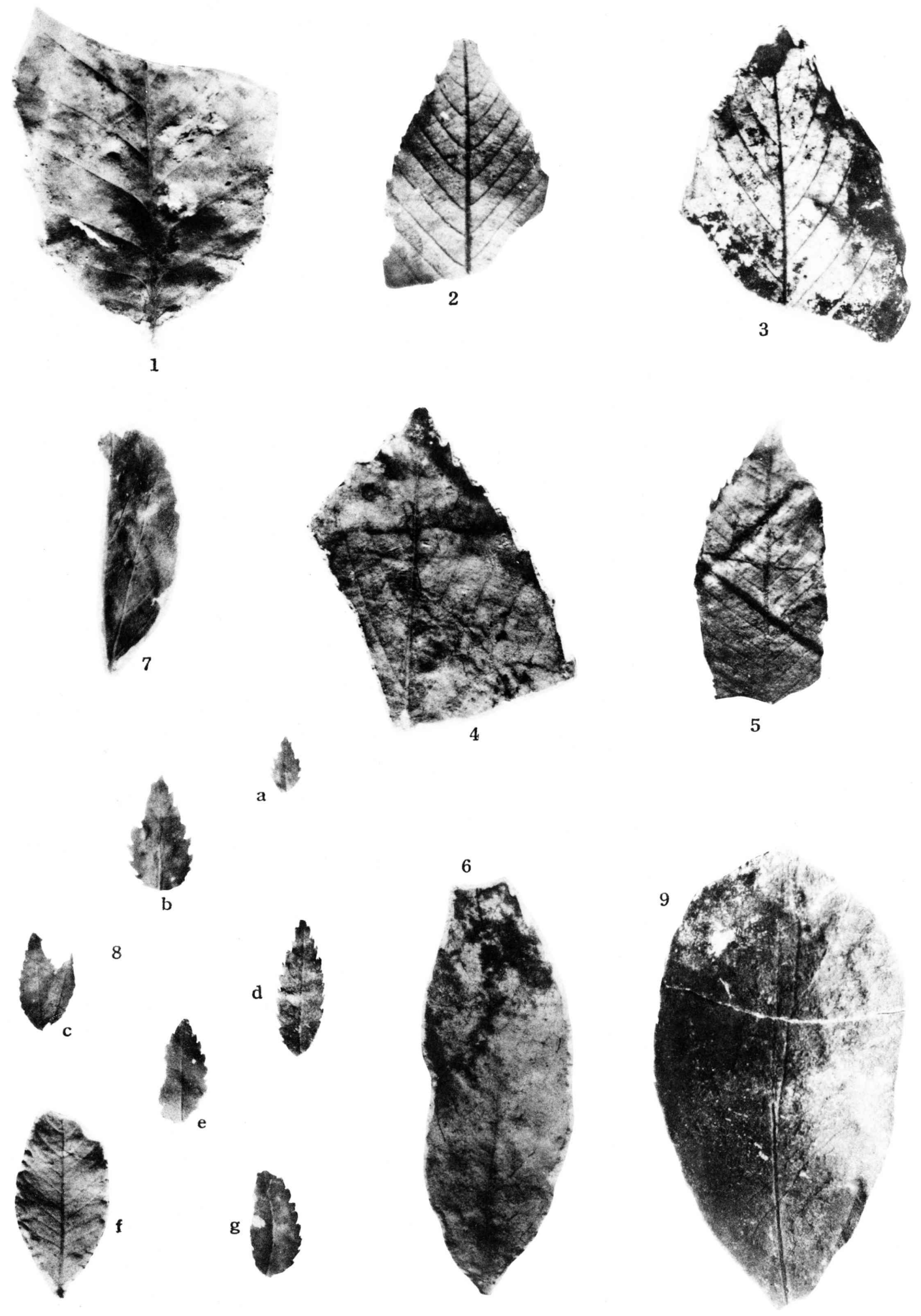


\section{Erklärung der Tafel VI}

Fig. 1 a-i. Entada formosana KANEHIRA

Fig. 2. Cinnamomum cf. Camphora Sieb.

Fig. 3. Cinnamomum sp.

Fig. 4. Liquidambar cf. formosana HANOE

Fig. $5 \mathrm{a}-\mathrm{m}$. Rosa multiflora THUNв.

$\mathrm{a}, \mathrm{b}, \mathrm{e}-\mathrm{m}$ : Blätter ; c, d: Dorne.

Fig. $6 \mathrm{a}, \mathrm{b}, \mathrm{c}$. Rosa $\mathrm{sp}$.

Fig. 7 a, b. Wistaria cf. brachybotys S. et Z. (?)

Die Objekte sind in natürlicher Grösse gezeichnet. 

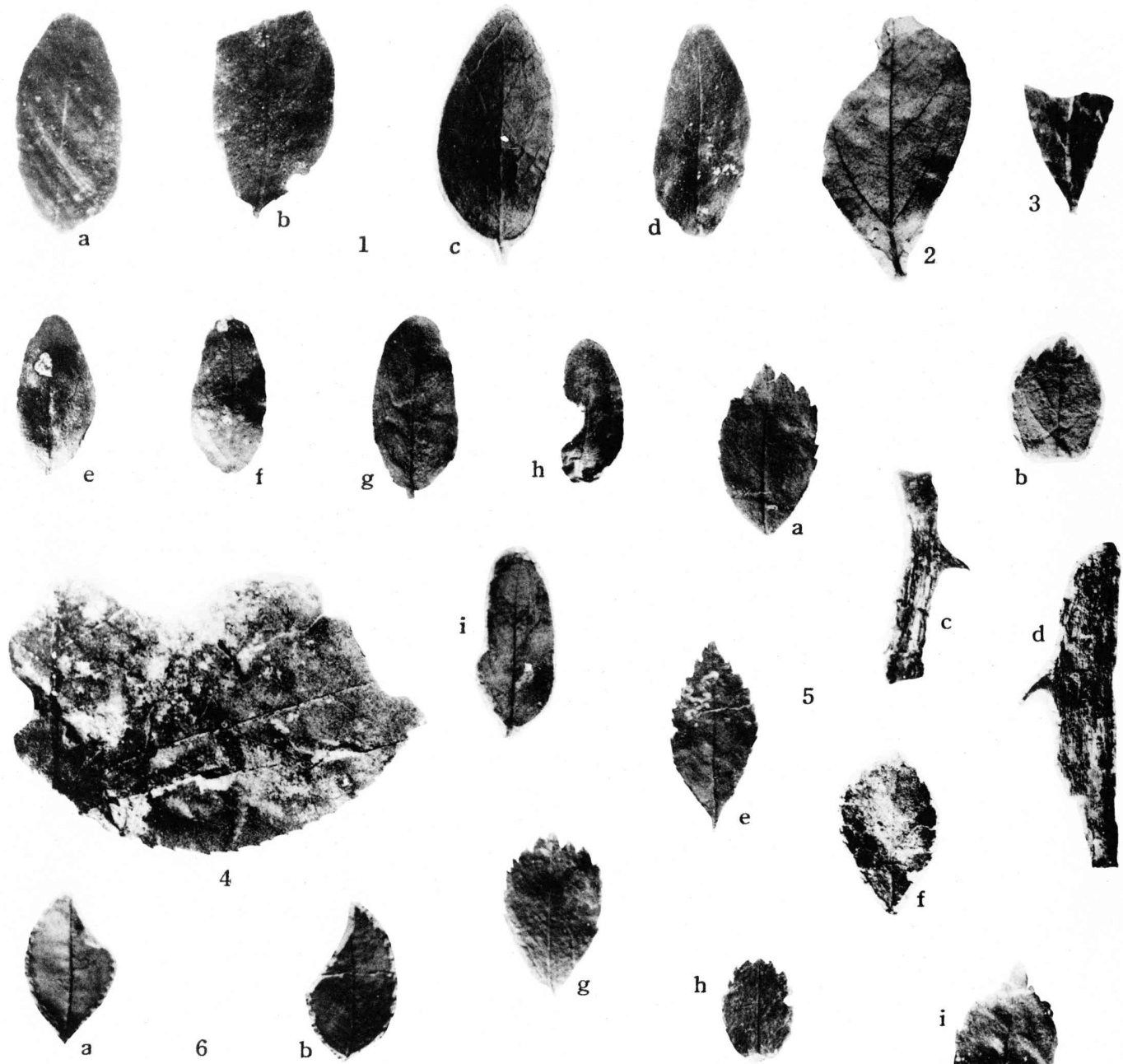

b

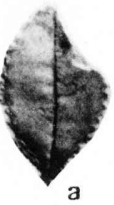

6
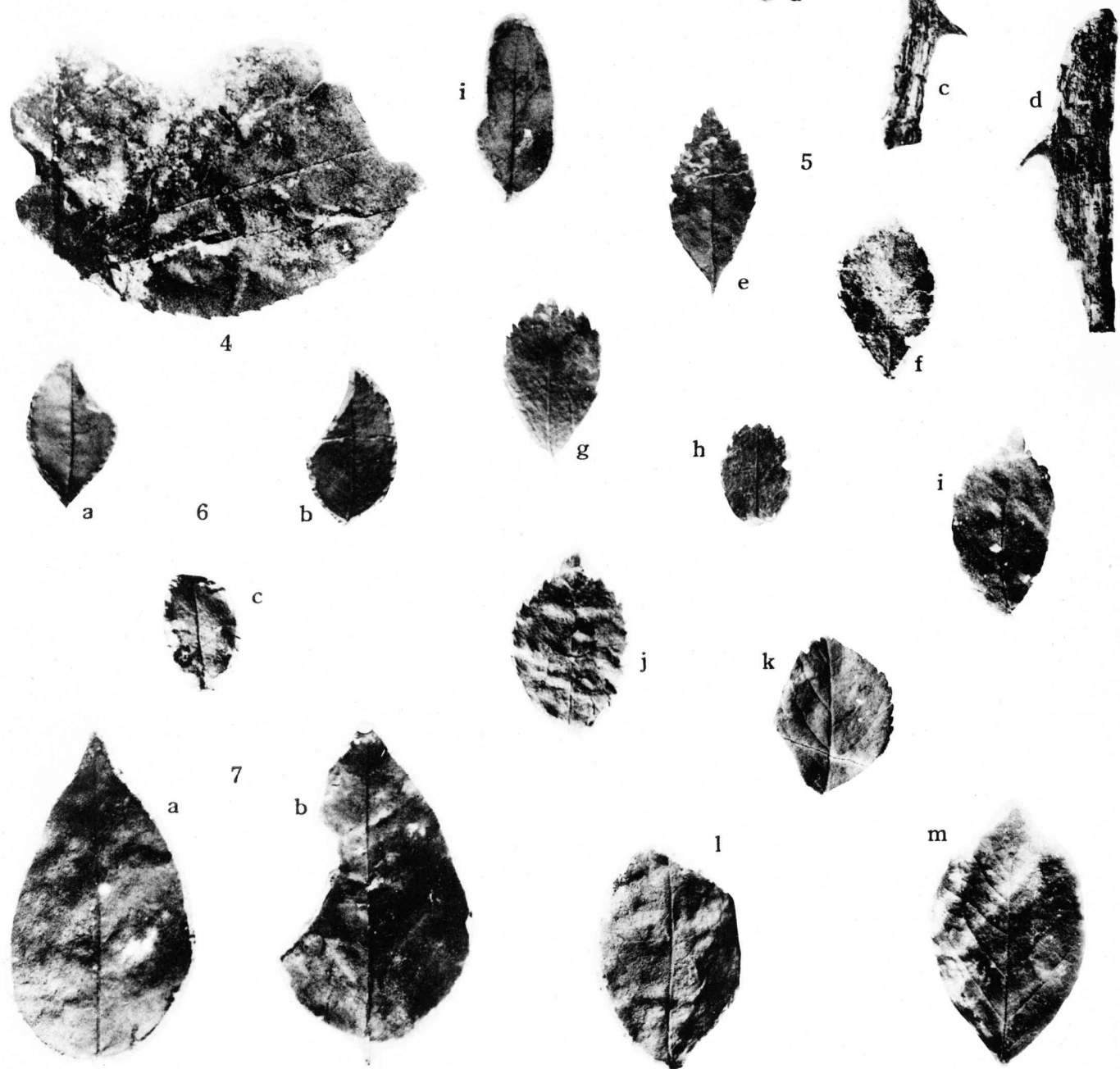


\section{Erklärung der Tafel VII}

Fig. 1 a, b. Acer cf. parviflorum F. et S. (?)

Fig. 2. Acer cf. diabolicum B.. (?)

Fig. 3, 4 a, b. Acer pictum ThunB.

Fig. 5, 6, 7 a, b. Acer palmatum Thuns.

Fig. 8 a-d. Sapium sebiferum Rox. var. pleistoceaca MIKI

Fig. 9. Tilia cf. japonica Siмк.

Fig. 10. Xanthoceras cf. sorbifolia BGE.

Fig. 11 a-d. Paliurus nipponicus MrkI

Fig. 12. Stewartia cf. monadelpha S. et Z.

Fig. $13 \mathrm{a}-\mathrm{g}$. Buxus japonica Muell

Die Objekte sind in natürlicher Grösse gezeichnet. 


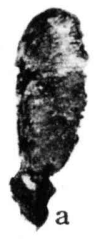

1

b
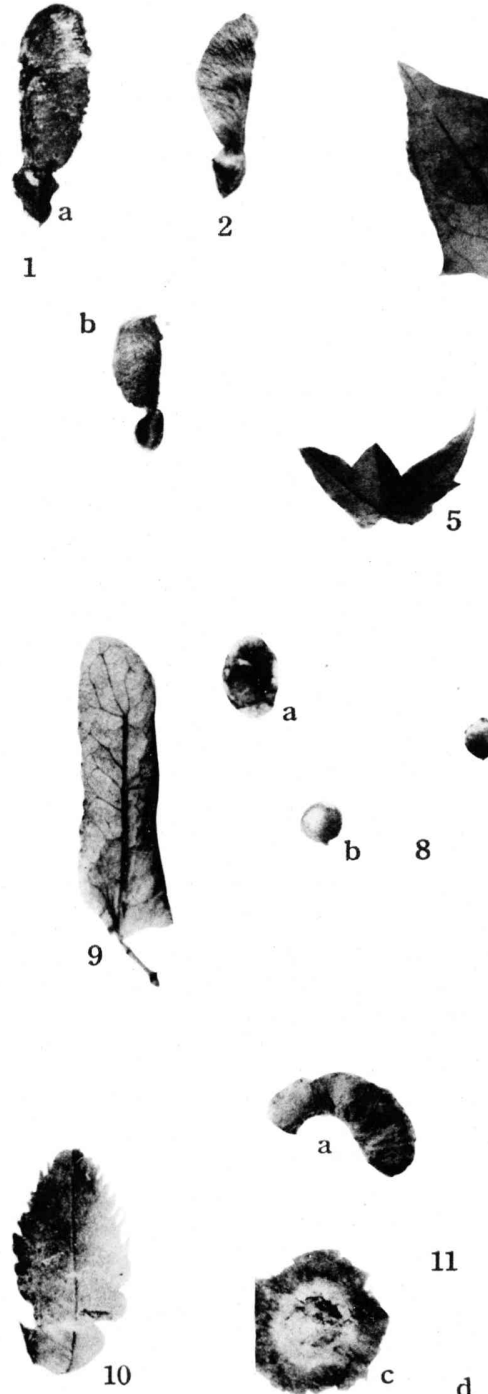

is
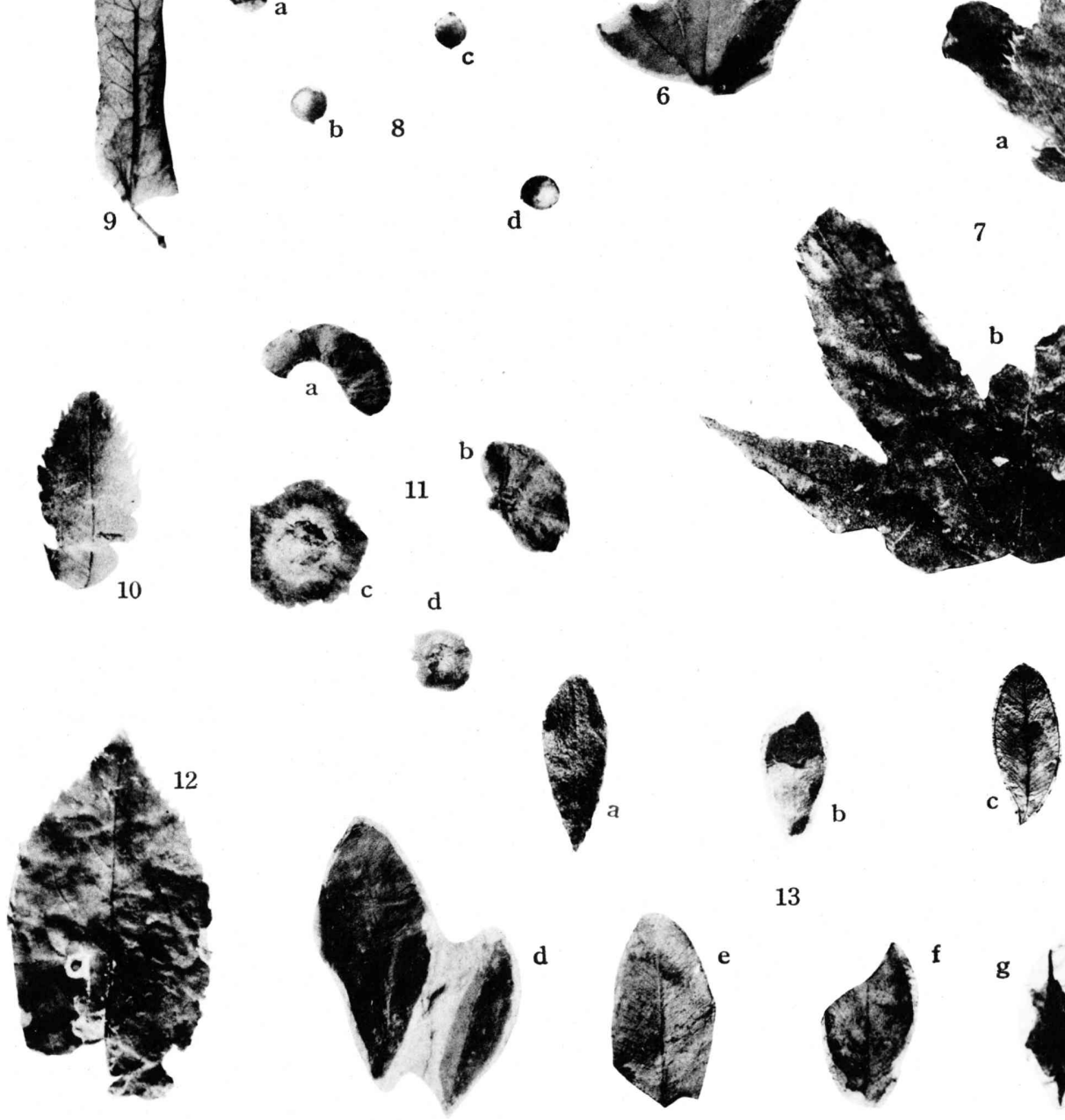

13
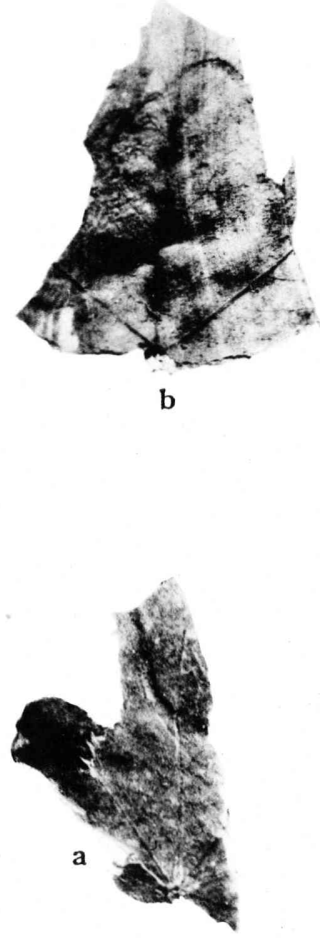

7

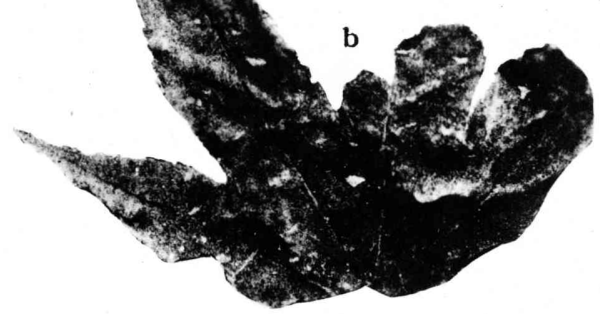

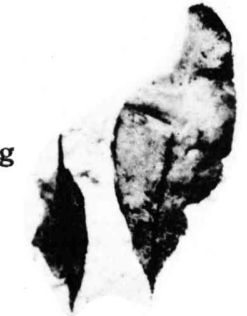




\section{Erklärung der Tafel VIII}

Fig. 1 a-m. Smilax China L.

Fig. 2 a, b, c. Camellia japonica L.

Fig. 3. Diospyros Kaki Thunb.

Die Objekte sind in natürlicher Grösse gezeichnet. 

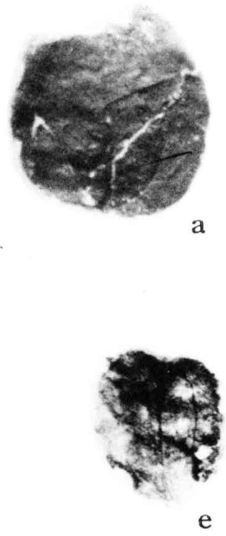
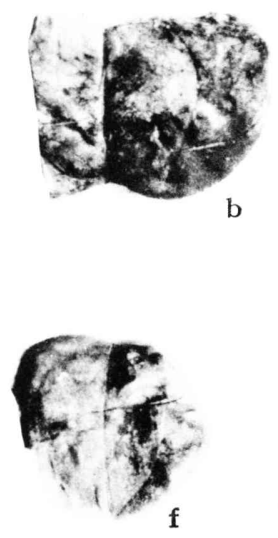

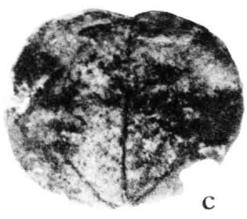

1

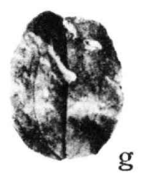

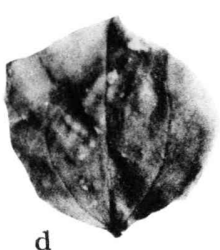

d

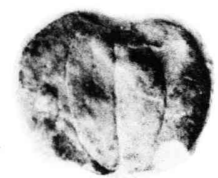

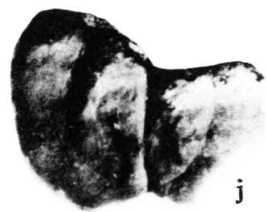
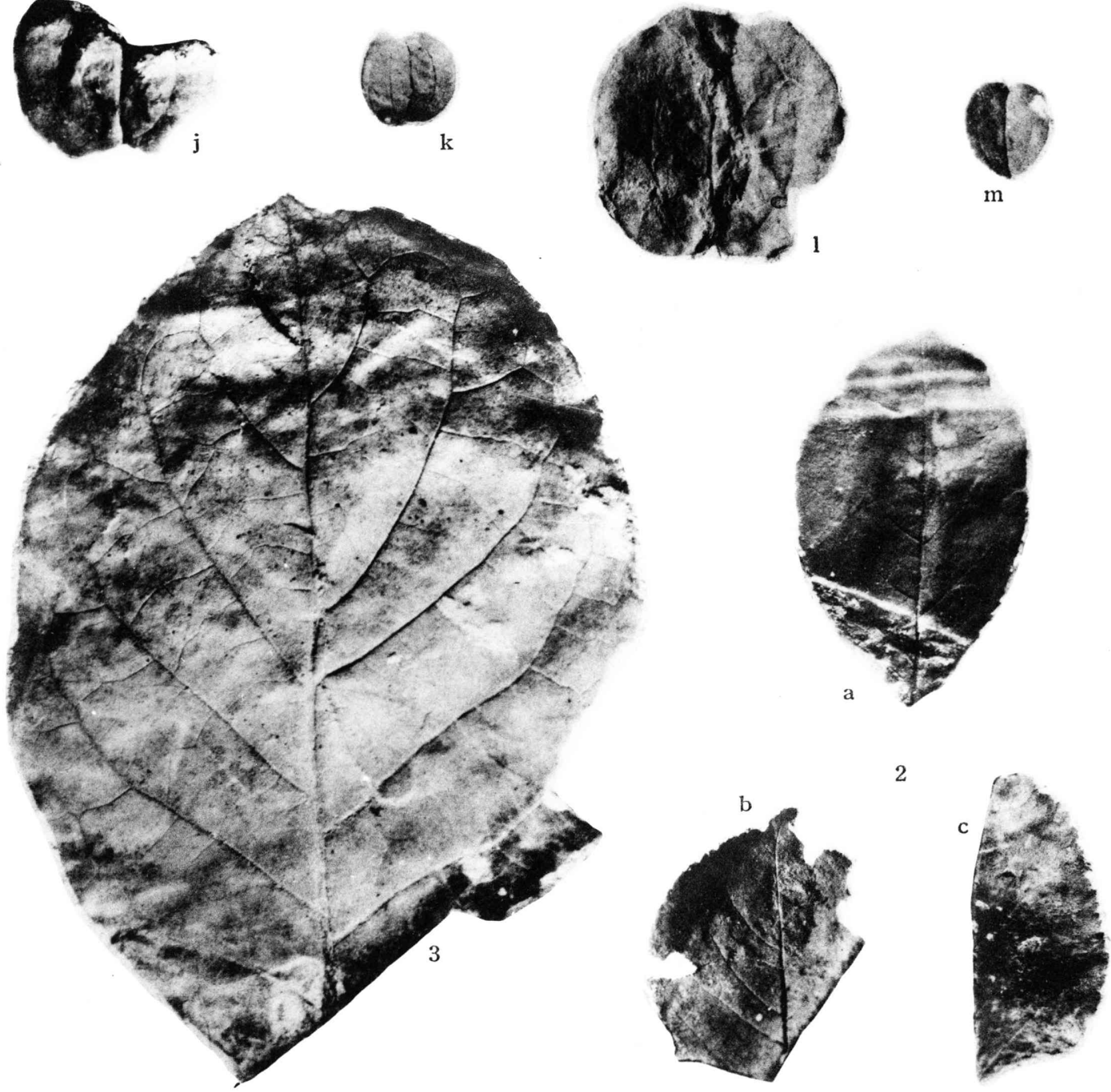